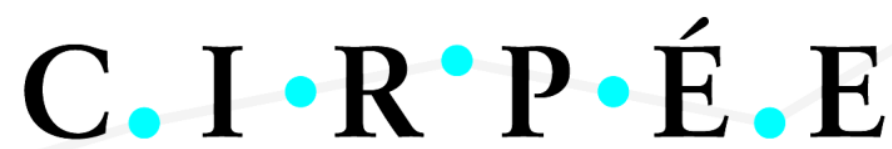 \\ Centre Interuniversitaire sur le Risque, les Politiques Économiques et l'Emploi
}

Cahier de recherche/Working Paper 10-18

\section{Strategic Interaction and Networks}

\author{
Yann Bramoullé \\ Rachel Kranton \\ Martin D'Amours
}

Mai/May 2010

\footnotetext{
Bramoullé: Département d'économique, Université Laval and CIRPÉE ybramoulle@ecn.ulaval.ca

Kranton: Duke University

D’Amours: Département d'économique, Université Laval

We dedicate this paper to the memory of Toni Calvó-Armengol. As will be clear in the subsequent pages, he has made a lasting contribution to our thinking about networks and economics. We deeply miss his insight and his company. We are grateful to Alexander Groves for research assistance and to Noga Alon, Roland Bénabou, Francis Bloch, Andrea Galeotti, Sanjeev Goyal, Matthew Jackson, Antoine Loeper, Brian Rogers, and Yves Zenou and participants in seminars and conferences at Barcelona, Berkeley, Caltech, Cambridge, Duke, École Polytechnique (Paris), Essex, MEDS (Northwestern), Laval (Québec), Montréal, NYU Stern School, Paris School of Economics, and Stockholm for helpful comments and discussions. For support, Rachel Kranton thanks the Canadian Institute for Advanced Research, and Yann Bramoullé thanks the Social Sciences and Humanities Research Council and the Canada Research Chair in Social Policies and Human Resources.
} 


\begin{abstract}
:
This paper brings a general network analysis to a wide class of economic games. A network, or interaction matrix, tells who directly interacts with whom. A major challenge is determining how network structure shapes overall outcomes. We have a striking result. Equilibrium conditions depend on a single number: the lowest eigenvalue of a network matrix. Combining tools from potential games, optimization, and spectral graph theory, we study games with linear best replies and characterize the Nash and stable equilibria for any graph and for any impact of players' actions. When the graph is sufficiently absorptive (as measured by this eigenvalue), there is a unique equilibrium. When it is less absorptive, stable equilibria always involve extreme play where some agents take no actions at all. This paper is the first to show the importance of this measure to social and economic outcomes, and we relate it to different network link patterns.
\end{abstract}

Keywords: Networks, potential games, lowest eigenvalue, stable equilibria, asymmetric equilibria

JEL Classification: C72, D00 


\section{Introduction}

In many economic settings, people and firms do not interact equally and directly with everyone. A network, or interaction matrix or graph, can formally capture various cross-cutting links and relationships by indicating whose actions directly affect whose payoffs. The matrix can represent social links, geography, agents' preferences, market structure, or information flows. Because linked players interact with other linked players, outcomes depend on the entire network structure. The major challenge and interest is determining how network structure shapes outcomes. Networks are complex objects and answering this question is generally difficult even in otherwise simple settings. We study strategic interaction and networks for an important class of games - games with linear best replies. Such games have been used to study a wide variety of settings including public goods, oligopoly, resource extraction, criminal activity, investment, belief formation, and peer effects. ${ }^{1}$ We bring to bear a new combination of tools and characterize the Nash and stable equilibria for any graph structure and for any impact of players' actions on others' payoffs.

We have a striking result. Using tools from potential games, optimization, and spectral graph theory, we find that equilibrium outcomes depend on a single number: the lowest eigenvalue of a network matrix. When the network matrix is sufficiently absorptive (as measured by this eigenvalue), there is a unique equilibrium. An equilibrium is stable when the graph connecting active agents is sufficiently absorptive (as measured by the lowest eigenvalue). This paper is the first to show the importance of this measure to social and economic outcomes, and we relate it to patterns of network links. ${ }^{2}$

While our findings hold in general, we focus on games of strategic substitutes. Strategic substitutes settings include oligopoly interaction, public good provision, research \& development, price and information-gathering by consumers, and experimentation with new technologies. ${ }^{3}$ Strategic substitute games involve complexities not present with strategic complements. Agents' actions tend to go in opposite directions. There are often multiple equilibria, which cannot generally be neatly ordered. To tackle multiple equilibria, we consider stable equilibria as a subset of Nash

\footnotetext{
${ }^{1}$ See, for example, Angeletos \& Pavan (2007), Ballester, Calvó-Armengol \& Zenou (2006), Bergemann \& Morris (2009), Calvó-Armengol, Patacchini \& Zenou (2009), Bénabou (2008), Bramoullé \& Kranton (2007), İlkiliç (2008), Glaeser \& Scheinkman (2003), Goyal \& Moraga-González (2001), and Vives (1999).

${ }^{2}$ For a compendium of network measures, see Wasserman \& Faust (1994) and Scott (2004). Echenique \& Fryer (2007), for example, use the highest eigenvalue as a measure of segregation.

${ }^{3}$ See, e.g., Vives (1999), Foster \& Rosensweig (1995), Gladwell (2000), and Grabher (1993).
} 
equilibria. Stable equilibria are robust to small changes in agents' play. ${ }^{4}$ We find an equilibrium is stable when the graph connecting active agents has large absorptive capacity (measured by the lowest eigenvalue) relative to the impact of play. We also find that, except for small payoff impacts, stable equilibria always involve asymmetric and extreme outcomes - some players do nothing at all. Thus the common restriction of attention in the literature to symmetric equilibria can be misleading. To tackle comparative statics, we use our combination of tools to show when for stable equilibria, and overall, aggregate actions decrease. Thus, for example, an additional link in a network can decrease the total provision of public goods. ${ }^{5}$

From these results the paper builds general intuitions about strategic play in networks. First, even when agents have symmetric network positions, play is likely to be concentrated on a few players. Interior and symmetric outcomes are the exception rather than the norm. Second, while there is a rich variety of equilibria, there is a tight relation between the structure of the equilibrium set and the shape of the overall network. The lowest eigenvalue gives a critical measure of this relation. Third, this mathematical index relates to the geometry of the network. It captures partitions of society into distinct sets. When individuals have fewer links within sets and more links between sets, the system tends to amplify payoff impacts and to reach corner outcomes.

This paper makes at least three contributions.

First, this paper advances the analysis of $n$-person simultaneous-move games with continuous action spaces. As mentioned above, there is a wide variety of applications that are modeled with payoffs that have linear best responses. The canonical game is one with quadratic payoffs. Often researchers restrict attention to (a) the case where all agents interact equally with all other agents, (b) symmetric equilibria, and (c) interior equilibria. This paper gives the tools to study any possible interaction structure, the entire action space, and the full set of equilibrium outcomes. ${ }^{6}$ New conclusions are then possible, such as comparative statics and how equilibria relate to network structure.

Second, we advance the recent and active literature on games played on networks. This paper

\footnotetext{
${ }^{4}$ Formally, we consider asymptotically stable equilibria. Given a system of linear differential equations defined using agents' best response functions, an equilibrium is stable when the system converges back to the equilibrium following any small enough perturbation in agents' actions.

${ }^{5}$ Bramoullé \& Kranton (2007) contains a special case of this result, and Bloch \& Zenginobuz (2007) show spillovers can lead to lower public good provision.

${ }^{6}$ For instance, our approach can be used to study the leading public goods example in Bergemann \& Morris $(2008,2009)$ for an arbitrary network.
} 
integrates and supercedes findings in two main lines of inquiry. In this previous work, games have quite different payoff functions, but - as we point out here-best-response functions are identical and linear. Ballester, Calvó-Armengol \& Zenou (2006) study a quadratic-payoff game where agents are harmed by other agents' actions. They examine outcomes when agents have weak impacts on others, identifying a sufficient condition for a unique, interior, equilibrium. In this equilibrium agents' actions are proportional to their Bonacich centrality measures. ${ }^{7}$ Bramoullé \& Kranton (2007) study a game where agents gain from other agents' actions. The analysis examines the opposite end of the spectrum, when agents have strong impacts on others (actions are perfect substitutes). Multiple equilibria are the rule, and there is always an equilibrium where some individuals choose an action of zero. These specialized equilibria involve maximal independent sets of the network graph. The present paper analyzes the full range of impacts - agents' impacts on others ranges from weak to strong - for any graph. We identify a precise relationship between graph structure, payoff impacts, and unique and stable equilibria.

With these findings, we also make significant progress on the problems of multiplicity of equilibria and comparative statics in network settings. Researchers in this area are often frustrated by the large number of equilibria and the difficulty of characterizing and comparing equilibria. Galeotti, Goyal, Jackson, Vega-Redondo \& Yariv (2009) explore how to overcome these issues by assuming players have limited information about the graph structure. They obtain sharp analytical results for symmetric equilibria. In the present paper, we take a more traditional approach; agents play a simultaneous move game on a graph, and we explore Nash and stable equilibria. To attack characterizing equilibria and comparative statics, we use different tools and concepts such as potential functions and structural network features.

Third, the paper connects traditional economics techniques and outcomes to traditional computer science. We characterize equilibrium outcomes as solutions to well-defined quadratic programming problems. We construct an (necessarily exponential time) algorithm to identify all equilibria and show that solving for certain focal equilibria is directly related to a well-known NP hard problem. We exploit Monderer \& Shapley's (1996) theory of potential games that computer scientists have used to study congestion and other issues (e.g., Roughgarden \& Tardos (2002),

\footnotetext{
${ }^{7}$ Ballester, Calvó-Armengol \& Zenou (2006) are the first to make the connection between centrality measures and strategic play. In a similar game, Ballester \& Calvó-Armengol (2007) find a sufficient condition for a unique equilibrium. Corbo, Calvó-Armengol \& Parkes (2007) study network design in the same setting.
} 
Chien \& Sinclair (2007)). In economics, the theory of potential games has been applied to finite games, including a few network settings. ${ }^{8}$ Neyman (1997) studies potential games with continuous actions and finds conditions for a unique correlated equilibrium. We provide the first application to network games with a continuous action space. We show how to use it, in combination with other tools, to illuminate strategic interaction in networks. These cross-overs could have great impact on overall study of networks and multiple player games.

The paper proceeds as follows. In the next section we present the model and give our basic characterization of Nash equilibria. Section III finds the relationship between the lowest eigenvalue of the network matrix and unique and corner equlibria. We study stable equilibria in Section IV. Section V connects the lowest eigenvalue to network link patterns. We conduct comparative statics in Section VI. We show how our results generalize and apply to many more games in Section VII and conclude in Section VIII.

\section{Class of Games and Nash Equilibria}

\section{A. The Model}

We present the basic model here. The results extend generally as described in Section VII.

There is a set of $n$ agents denoted $N$. Each agent $i$ simultaneously chooses an action $x_{i} \in$ $[0, \infty)$. Let $\mathbf{x}$ denote an action vector for all agents and $\mathbf{x}_{-i}$ denote an action vector of all agents other than $i$. To specify payoffs, we use a graph, or network, or interaction matrix, which is an $n \times n$ matrix $\mathbf{G}=\left[g_{i j}\right],{ }^{9}$ where $g_{i j}=1$ if $i$ impacts $j$ 's payoffs directly, $g_{i j}=0$ otherwise, and it is assumed $g_{i j}=g_{j i} \cdot{ }^{10}$ We set $g_{i i}=0$. Following common usage, we say agent $i$ and $j$ are linked, connected, or neighbors when $g_{i j}=g_{j i}=1$. Payoffs are

$$
U_{i}\left(x_{i}, \mathbf{x}_{-i} ; \delta, \mathbf{G}\right)
$$

where $\delta \in \mathbb{R}$ is the payoff impact parameter; i.e., how much $i$ 's neighbors affect $U_{i}$. Let $\bar{x}_{i}$ be the action individual $i$ would take in isolation; $\bar{x}_{i}$ maximizes $U_{i}$ when $\delta=0$ or $g_{i j}=0$ for all $j$.

\footnotetext{
${ }^{8}$ Blume (1993) studies finite games when players are situated on a lattice. Young (1998) considers coordination games with two actions and general networks. Bramoullé (2007) studies anti-coordination games with two actions and general networks.

${ }^{9}$ Throughout the paper, we identify a network with its adjacency matrix.

${ }^{10}$ The analysis applies to any weighted graph with $g_{i j}=g_{j i} \in \mathbb{R}$ as discussed in Section VII.
} 
For the basic model, $\delta \in[0,1]$ which, in combination with $g_{i j} \geq 0$, will yield strategic substitutes. Our results apply to the less complicated setting of strategic complements, where $\delta \leq 0$ or $g_{i j} \leq 0$, contained in Section VII, along with mixed strategic substitutes and complements. ${ }^{11}$

\section{B. Class of Games: Payoffs with Linear Best Replies}

We study games where payoff functions have different functional forms but all have the same linear best reply functions. As discussed, canonical games in economics and the network literature fall in this class. We find pure-strategy Nash and stable equilibria. ${ }^{12}$ Since the games have the same best reply functions, the games have the same set of equilibrium outcomes.

We first present three exemplary games in this class. We then fully describe the best-reply functions. In each of the games that follow $\bar{x}_{i}=\bar{x}$ for all $i$. This will be the base case, and the results hold generally as discussed in Section VII.

The first game is a model of public goods in networks (Bramoullé \& Kranton (2007)). Individual $i$ benefits from his own and his neighbors' actions, and individual $i$ 's payoff is

$$
\widehat{U}_{i}\left(x_{i}, \mathbf{x}_{-i} ; \delta, \mathbf{G}\right)=b\left(x_{i}+\delta \sum_{j} g_{i j} x_{j}\right)-\kappa x_{i}
$$

where $b($.$) is strictly increasing and strictly concave on \mathbb{R}_{+}$, and $\kappa>0$ is the constant marginal cost of own action, such that $b^{\prime}(0)>\kappa>b^{\prime}(+\infty)$. As in Bergstrom, Blume \& Varian (1986), public goods are privately provided, but here public goods are local. Bramoullé \& Kranton (2007) primarily study the case of $\delta=1$ ( $i$ 's neighbors' actions are perfect substitutes for $x_{i}$ ).

The second game is from Ballester, Calvó-Armengol \& Zenou (2006) where agents are hurt, not helped, by neighbors. Individual $i$ 's payoff is

$$
\widetilde{U}_{i}\left(x_{i}, \mathbf{x}_{-i} ; \delta, \mathbf{G}\right)=\bar{x} x_{i}-\frac{1}{2} x_{i}^{2}-\delta \sum_{j} g_{i j} x_{i} x_{j}
$$

For future reference, we call $\widetilde{U}_{i}$ quadratic payoffs. Ballester, Calvó-Armengol \& Zenou (2006) and

\footnotetext{
${ }^{11}$ Thus our analysis readily accomodates local complementarities and global substitutabilities as in Ballester, Calvó-Armengol \& Zenou's (2006) general model. The graph G would give the net of the complements and substitutes for each $i j$. That is, $\mathbf{G} \equiv \gamma \mathbf{C}-\theta \mathbf{L}$, where $\gamma$ and $\theta$ are positive parameters, $\mathbf{C}$ is the complete graph, and in graph $\mathbf{L} l_{i j}=l_{j i} \geq 0$ for all $i j$ and $l_{i i}=0$ for all $i$.

${ }^{12}$ For games in the class, agents never have an incentive to randomize, hence restriction to pure strategies is without loss of generality.
} 
Ballester \& Calvó-Armengol (2007) study this game for $\delta$ low.

The third game is a standard differentiated-product Cournot oligopoly with linear (inverse) demand and constant marginal cost. ${ }^{13}$ With marginal cost $d$, firm $i$ facing demand $P_{i}\left(x_{i}, \mathbf{x}_{-i} ; \delta, \mathbf{G}\right)=$ $a-b \cdot\left(x_{i}+2 \delta \sum_{j} g_{i j} x_{j}\right)$ has payoffs:

$$
\Pi_{i}\left(x_{i}, \mathbf{x}_{-i} ; \delta, \mathbf{G}\right)=x_{i}\left(a-b \cdot\left(x_{i}+2 \delta \sum_{j} g_{i j} x_{j}\right)\right)-d x_{i}
$$

where $g_{i j}=1$ indicates $i$ and $j$ 's products are substitutes and $\delta$ captures the degree of substitutability. Vives (1999) considers interior equilibria.

In these three games agent $i$ has the same best response function:

$$
\begin{array}{lll}
x_{i}=\bar{x}-\delta \sum_{j} g_{i j} x_{j} & & \text { if } \delta \sum_{j} g_{i j} x_{j}<\bar{x} \quad \text { and } \\
x_{i}=0 & & \text { if } \delta \sum_{j} g_{i j} x_{j} \geq \bar{x} .
\end{array}
$$

Agent $i$ will achieve $\bar{x}$ through his own and his neighbors' actions. If the weighted sum of neighbors' actions is less than $\bar{x}$, agent $i$ makes up the difference. If the weighted sum is higher than $\bar{x}$, agent $i$ chooses 0 . Without loss of generality, normalize $\bar{x} \equiv 1$ to yield the best reply function $f_{i}(\mathbf{x}) \in[0,1]$ :

$$
f_{i}(\mathbf{x}, \delta, \mathbf{G})=\max \left(0,1-\delta \sum_{j=1}^{n} g_{i j} x_{j}\right) .
$$

We solve for the Nash equilibria and stable equilibria for games with this best-reply. A Nash equilibrium is a vector $\mathbf{x} \in[0,1]^{n}$ that simultaneously satisfies $x_{i}=f_{i}(\mathbf{x}, \delta, \mathbf{G})$ for all agents $i \in N$. A Nash equilibrium could be unstable in the sense that a small change in actions could leadthrough an adjustment process - to a different action vector. We study a continuous adjustment process and find asymptotically stable equilibria. ${ }^{14}$

Our objective is to solve for and characterize all Nash equilibria and all stable equilibria as they depend on the payoff parameter $\delta$ and on the graph G. Throughout the paper, for a given

\footnotetext{
${ }^{13}$ Linear inverse demand functions derive from consumers with strictly concave quadratic utility functions (Vives (1999)).

${ }^{14}$ We thus follow early work on Cournot games with multiple sellers (Fisher (1961)) that shows existence of stable equilibria with continuous but not discrete adjustment - a finding replicated here.
} 
graph $\mathbf{G}$ we say that a property holds for almost every $\delta$ if it holds for every $\delta$ except possibly a finite number of values. ${ }^{15}$

We use the following terminology and notation. We say a vector $\mathbf{x}$ is interior if $\forall i, x_{i}>0$. A vector $\mathbf{x}$ is a corner if for some agent $i, x_{i}=0$. A corner vector $\mathbf{x}$ is specialized if $\forall i, x_{i} \in\{0,1\}$. Let $\mathbf{I}$ denote the $n$-square identity matrix, let $\mathbf{1}$ denote a vector of ones, $\mathbf{o}$ a vector of zeros, and $\mathbf{O}$ a square matrix of zeros. The empty network is then $\mathbf{G}=\mathbf{O}$. In a complete network, denoted by $\mathbf{C}$, every agent is connected to every other agent.

For individual agents, let $k_{i}$ denote the number of $i$ 's neighbors, called $i$ 's degree; $k_{i}=\sum_{j} g_{i j}$. Let $k_{\min }(\mathbf{G})$ and $k_{\max }(\mathbf{G})$ respectively, denote the smallest and largest degree in graph $\mathbf{G}$. For an agent's centrality, given a graph $\mathbf{G}$ and a scalar $q$ such that $\mathbf{I}-q \mathbf{G}$ is invertible, the vector of Bonacich centralities $\mathbf{c}(q, \mathbf{G})$ is defined by $\mathbf{c}(q, \mathbf{G})=(\mathbf{I}-q \mathbf{G})^{-1} \mathbf{G} \mathbf{1}$ (Bonacich (1987)). The centrality measure for agent $i, c_{i}(q, \mathbf{G})$, can be seen as a weighted sum of the paths in $\mathbf{G}$ that start with $i .^{16}$

For overall graph structure, let $\lambda_{\min }(\mathbf{G})$ and $\lambda_{\max }(\mathbf{G})$ denote the lowest and highest eigenvalues of $\mathbf{G}$, respectively, and let $\rho(\mathbf{G}) \equiv \max \left(\left|\lambda_{\min }(\mathbf{G})\right|,\left|\lambda_{\max }(\mathbf{G})\right|\right)$ denote its spectral radius.

The value $\lambda_{\min }(\mathbf{G})$ features prominently in our results. Since any graph $\mathbf{G}$ is square and symmetric, $\lambda_{\min }(\mathbf{G}) \leq 0$ and $\lambda_{\max }(\mathbf{G}) \geq 0$. Furthermore, $\lambda_{\max }(\mathbf{G}) \geq-\lambda_{\min }(\mathbf{G}) .{ }^{17}$ For a higher $\lambda_{\min }(\mathbf{G})$, we say the graph has more absorptive capacity. We will see that an absorptive graph dampens - rather than magnifies - the impact of players' actions captured in $\delta$.

\section{Complete Set of Nash Equilibria}

Existence of Nash equilibria is guaranteed thanks to a fixed-point argument. ${ }^{18}$ The equilibrium set has been well-studied when $\delta$ is low (Ballester, Calvó-Armengol \& Zenou (2006)) and when $\delta=1$ (Bramoullé \& Kranton (2007)). Little has been known for the general case.

Our first proposition gives the general characteristics of pure strategy Nash equilibria for any $\delta$ and $\mathbf{G}$. For a vector $\mathbf{x}$, let $A$ denote the set of active agents - those agents whose actions are

\footnotetext{
${ }^{15}$ For a graph $\mathbf{G}$, the set of $\delta$ values for which the property does not hold has zero measure.

${ }^{16}$ When $|q|$ is sufficiently small, $\mathbf{c}(q, \mathbf{G})=\mathbf{G} \mathbf{1}+q \mathbf{G}^{2} \mathbf{1}+q^{2} \mathbf{G}^{3} \mathbf{1}+\cdots$, and paths of length $k$ are weighted by $q^{k-1}$.

${ }^{17}$ For any square matrix, the sum of the eigenvalues is equal to its trace. For any symmetric matrix, all eigenvalues are real numbers. Since $\mathbf{G}$ is a nonnegative matrix, $\lambda_{\max }(\mathbf{G}) \geq-\lambda_{\min }(\mathbf{G})$ by Perron's theorem (see Theorem 0.13 in Cvetković et al. (1979)).

${ }^{18}$ The best-response function $\mathbf{f}$ is continuous from $[0,1]^{n}$ to itself. By Brouwer's theorem, $\mathbf{f}$ has a fixed point.
} 
strictly positive: $A=\left\{i: x_{i}>0\right\}$. Agents outside this set are called inactive agents. Let $\mathbf{x}_{A}$ denote the action vector of active agents; let $\mathbf{G}_{A}$ denote the subgraph of $\mathbf{G}$ connecting the active agents; and let $\mathbf{G}_{N-A, A}$ denote the graph of links connecting active agents to inactive agents. Parsing the network in this way and using matrix notation to express the best-reply conditions in (2) leads directly to the following result.

Proposition 1. A profile $\mathbf{x}$ with active agents $A$ is a Nash equilibrium if and only if

$$
\begin{aligned}
\left(\mathbf{I}+\delta \mathbf{G}_{A}\right) \mathbf{x}_{A} & =\mathbf{1} \\
\delta \mathbf{G}_{N-A, A} \mathbf{x}_{A} & \geq \mathbf{1}
\end{aligned}
$$

From Proposition 1 we have several direct findings. ${ }^{19}$ First, we can compose a simple algorithm to find all the equilibria for any graph $\mathbf{G}$ and almost every $\delta$ : Given a graph $\mathbf{G}$, consider a subset $S \subset N$. If $\operatorname{det}\left(\mathbf{I}+\delta \mathbf{G}_{S}\right) \neq 0$, compute the profile $\mathbf{x}_{S}=\left(\mathbf{I}+\delta \mathbf{G}_{S}\right)^{-1} \mathbf{1}$ and set $\mathbf{x}_{N-S}=\mathbf{o}$. Check whether $\mathbf{x}_{S} \geq 0$ and $\delta \mathbf{G}_{N-S, S} \mathbf{x}_{S} \geq \mathbf{1}$. If these two sets of linear inequalities are satisfied, $\mathbf{x}$ is an equilibrium. If either condition fails, it is not. Repeating this procedure for every subset of $N$ yields all the equilibria for $\mathbf{G}$ and almost every $\delta .{ }^{20}$ Second, for any graph $\mathbf{G}$ and almost every $\delta$, the equilibria are finite, ${ }^{21}$ since for a given graph $\mathbf{G}, \forall S, \operatorname{det}\left(\mathbf{I}+\delta \mathbf{G}_{S}\right) \neq 0$ for almost every $\delta$.

Third, we see how individual equilibrium play relates to graph position. Comparing two agents whose neighbors are nested sets, for $\delta<1$ the agent with the larger set of neighbors plays less. Intuitively, an agent who is linked to more outside sources can take a lower action. In addition, all equilibria for any $\mathbf{G}$ and almost every $\delta$ can be described by the vector of individual Bonacich centralities in $\mathbf{G}_{A}$. For an equilibrium $\mathbf{x}$, simply invert the matrix $\mathbf{I}+\delta \mathbf{G}_{A}$. We then have equilibrium actions $\mathbf{x}_{A}=\left(\mathbf{I}+\delta \mathbf{G}_{A}\right)^{-1} \mathbf{1}=\mathbf{1}-\delta \mathbf{c}\left(-\delta, \mathbf{G}_{A}\right)$, and for active player $i$, $x_{i}=1-\delta c_{i}\left(-\delta, \mathbf{G}_{A}\right) .{ }^{22}$ Thus, we validate here Bonacich's (1987) original insight on the impact

\footnotetext{
${ }^{19}$ See Appendix for proofs.

${ }^{20}$ Any such algorithm must run in exponential time since the number of equilibria is potentially exponential. In the language of computer science, our algorithm is "a total enumeration method," and researchers have been looking for "partial enumeration methods" for such problems (Murty \& Yu (1988)). We have derived several results (available upon request) that help identify the active set of agents, and thus could reduce computational time. First, using the fact that $f \circ f$ is increasing, we obtain a lower bound on individual actions in any equilibrium. This yields a computationally fast procedure to determine agents who are active in all equilibria. Second, for the vector $\mathbf{x}_{s}=\left(\mathbf{I}+\delta \mathbf{G}_{S}\right)^{-1} \mathbf{1}$, removing those agents for whom $x_{i} \leq 0$ can yield the set $A$ of active agents.

${ }^{21}$ Even when not finite, the equilibrium set has a well-defined mathematical structure. For any $\delta$ and $\mathbf{G}$ the set of equilibria is a finite union of compact convex sets.

${ }^{22}$ Write $\left(\mathbf{I}+\delta \mathbf{G}_{A}\right)^{-1}\left(\mathbf{I}+\delta \mathbf{G}_{A}\right) \mathbf{1}=\mathbf{1}$. Developing yields $\mathbf{x}_{A}+\delta \mathbf{c}\left(-\delta, \mathbf{G}_{A}\right)=\mathbf{1}$.
} 
of a negative scalar (here $-\delta$ ) on his measure. With a negative scalar, an agent's Bonacich centrality tends to be higher when linked to agents who themselves have few neighbors. These agents then choose lower actions.

Proposition 1 yields equilibria for all $\delta \in[0,1]$ and supercedes previous findings for low $\delta$ and $\delta=1$. We briefly review here the previous results, as written in the notation of the present paper.

Low $\delta$ : Using the theory of positive matrices, Ballester, Calvó-Armengol \& Zenou (2006, Theorem 1) show that if $\delta<1 /\left(1+\lambda_{\max }(\mathbf{C}-\mathbf{G})\right)$, there is a unique interior equilibrium where agents' actions can be described by their Bonacich centralities in the graph $\mathbf{C}-\mathbf{G}$ for the scalar $\delta /(1-\delta) .{ }^{23}$ For future reference, we label this critical value as $\underline{\delta}_{B C A Z} \equiv 1 /\left(1+\lambda_{\max }(\mathbf{C}-\mathbf{G})\right)$.

High $\delta$ : Using graph theory, Bramoullé \& Kranton (2007, Theorem 1) show for $\delta=1$, specialized equilibria exist for any non empty graph. The active players in these equilibria constitute a maximal independent set of the graph $\mathbf{G} \cdot{ }^{24}$ For $\delta<1$, specialized equilibria relate to maximal independent sets where inactive agents are connected to at least $1 / \delta$ agents in the set.

The following examples illustrate our results with canonical network structures and highlight the advances over previous work, showing the equilibria for the full range of $\delta$.
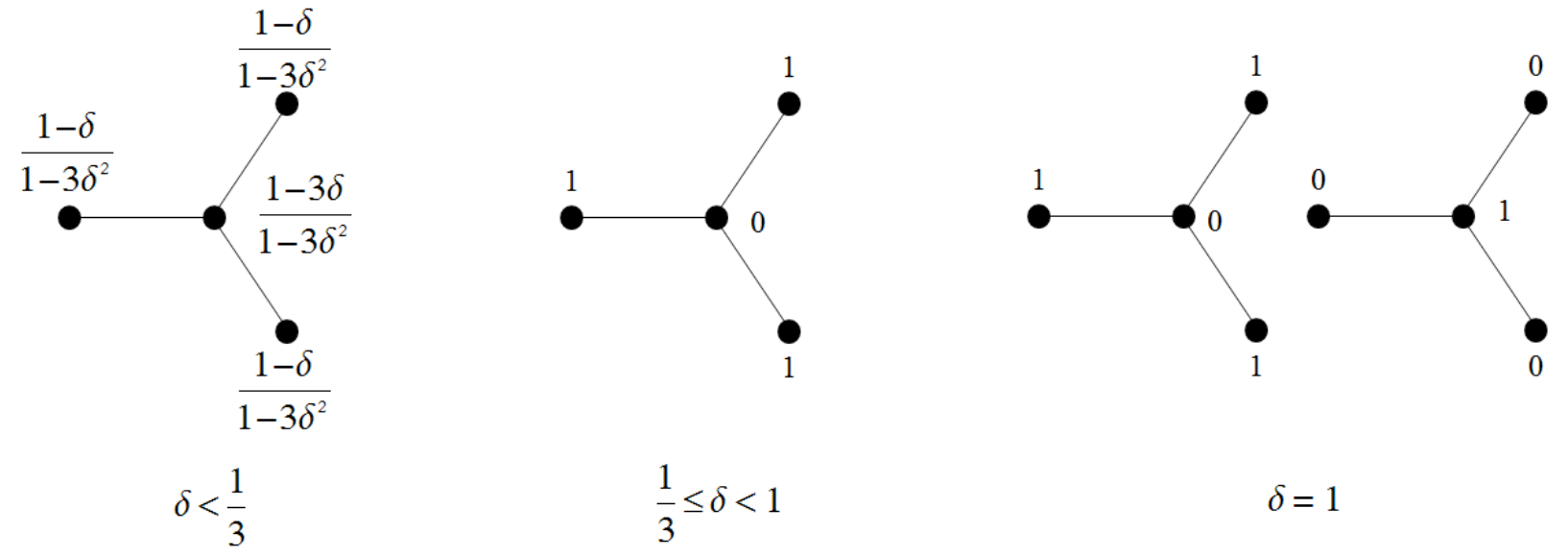

\footnotetext{
${ }^{23}$ Decomposing the network as $\mathbf{G}=\mathbf{C}-(\mathbf{C}-\mathbf{G})$, where $\mathbf{C}$ captures global substitutes and $(\mathbf{C}-\mathbf{G})$ captures local complements, and introducing the vector $\mathbf{y}=\mathbf{1}+\frac{\delta}{1-\delta} \mathbf{c}\left(\frac{\delta}{1-\delta}, \mathbf{C}-\mathbf{G}\right)$, their result states that if $\delta<1 /(1+$ $\left.\lambda_{\max }(\mathbf{C}-\mathbf{G})\right)$, the unique equilibrium action vector is $\mathbf{x}=\frac{1}{1-\delta+\delta \sum_{j} y_{j}} \mathbf{y}$, which is interior.

${ }^{24} \mathrm{~A}$ set of agents $I$ is an independent set of the graph $\mathbf{G}$ if for all $i, j \in I, g_{i j}=0$. An independent set $I$ is maximal if $I$ is not a subset of another independent set. Given any maximal independent set, agents in the population are divided into (i) those in the set and (ii) those linked to an agent in the set.
} 
Example 1. Stars. Consider a star with $n$ players. Figure 1, for $n=4$, shows, for $\delta<\underline{\delta}_{B C A Z}=$ $1 /(n-1)$ the unique, interior, equilibrium where the center plays $[1-(n-1) \delta] /\left[1-(n-1) \delta^{2}\right]$ and each peripheral agent plays $(1-\delta) /\left[1-(n-1) \delta^{2}\right]$. For $\delta=1$, there are two specialized equilibria: (i) the center plays 0 and peripheral agents play 1, and (ii) the center plays 1 and the peripheral agents play 0 . For the middle range, $1 /(n-1) \leq \delta<1$, there is a unique equilibrium. It is specialized; the center plays 0 and peripheral agents play 1. The star graph thus shows that unique equilibria can exist above $\underline{\delta}_{B C A Z}$, and the unique equilibrium need not be interior.

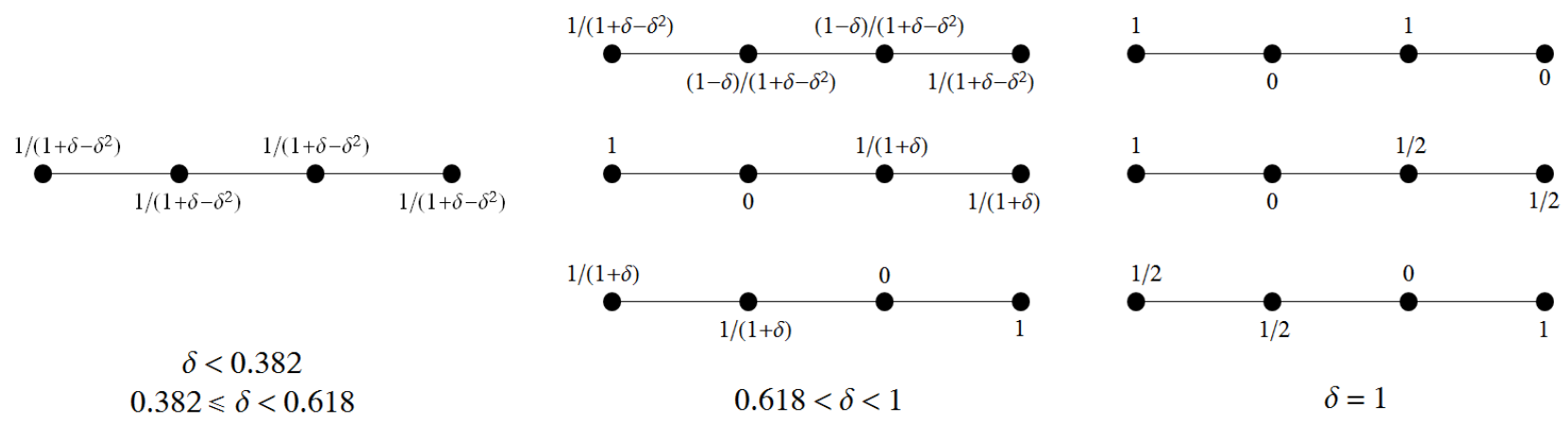

Figure 2: Equilibria in a Line Network

Example 2. Line with Four Players. Figure 2 shows for $\delta<\underline{\delta}_{B C A Z} \simeq 0.382$ the unique, interior, equilibrium where players 1 and 4 on the ends play $\frac{1}{1+\delta-\delta^{2}}$, and players 2 and 3 in the middle play $\frac{1-\delta}{1+\delta-\delta^{2}}$. For $\delta=1$, there are multiple equilibria, and the figure shows one specialized outcome where active agents are a maximal independent set of the graph. For the middle range of $\delta$, there is a unique equilibrium, which is interior, for $\underline{\delta}_{B C A Z}<\delta<\lambda_{\min }(\mathbf{G})=\frac{\sqrt{5}-1}{2} \simeq 0.618$. For $\frac{\sqrt{5}-1}{2}<\delta<1$, two corner equilibria emerge in addition to the interior one. To preview later results, in this range only the corner equilibria are stable. This example shows that an interior equilibrium can exist for any value of $\delta$, and it may not be unique or stable.

Example 3. Complex network. Figure 3 depicts equilibria on a complex network linking 20 agents. This network is a particular realization of a Poisson random graph with connection probability of 0.75. Agents with more links are depicted closer to the center, and a node's color is proportional to its action on a Black-White scale (Black=1, White=0). We see for $\delta<\underline{\delta}_{B C A Z} \simeq 0.153$, the unique equilibrium, which is interior. For the middle range of $\delta$, we show 


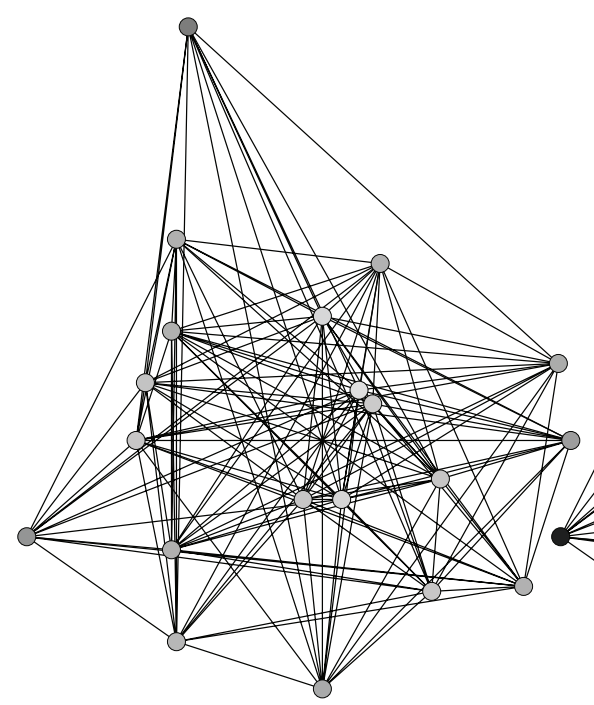

(a) $\delta=0.15$, unique equilibrium

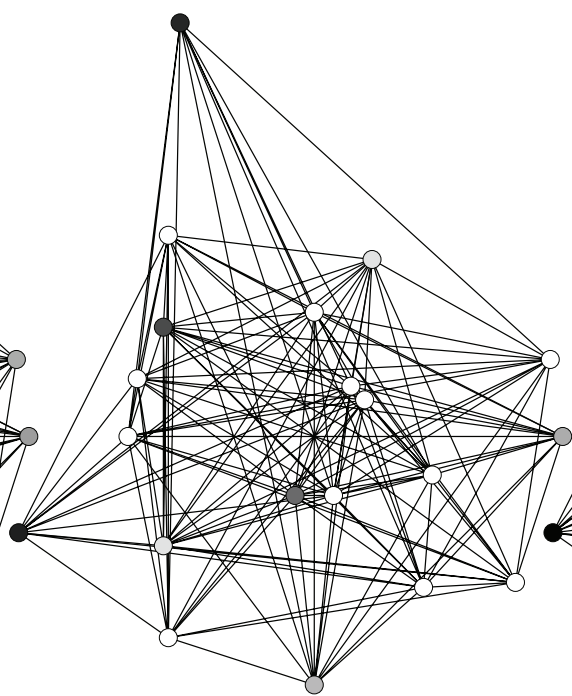

(b) $\delta=0.35,3$ equilibria

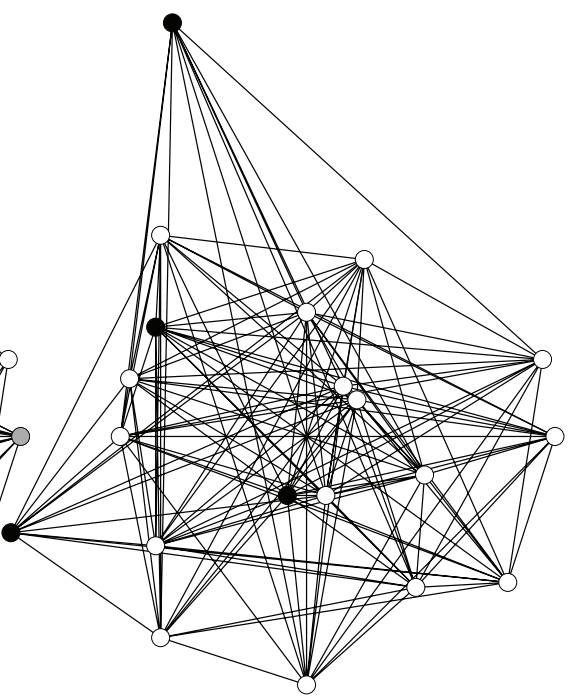

(c) $\delta=0.55,31$ equilibria

Figure 3: Equilibria in a Complex Network

one of the three equilibria for $\delta=0.35$ and one of the thirty-one equilibria for $\delta=0.55 .^{25}$ At $\delta=0.35$, many agents are inactive. At $\delta=0.55$, the equilibrium is specialized. This example shows the multiplicity and variety of equilibria for intermediate values of $\delta$.

\section{The Shape of Nash Equilibria}

We now address the major challenge: how network structure shapes equilibrium outcomes. We derive sufficient conditions and necessary conditions for unique, interior, corner, and stable outcomes - all as functions of the network structure and payoff parameter $\delta$. We also address comparative statics, a well-known problem in the analysis of strategic substitutes games. To do so, we combine tools from the theory of potential games, the theory of maximization, and spectral graph theory.

We find the lowest eigenvalue of a graph, $\lambda_{\min }(\mathbf{G})$, is key to equilibrium outcomes. This result is all the more remarkable because we also show that generally finding all equilibria is a computationally complex problem (requiring exponential time). We show that when a graph has a high $\lambda_{\min }(\mathbf{G})$, the equilibrium is unique. An equilibrium is stable if and only if $\lambda_{\min }\left(\mathbf{G}_{A}\right)$ is sufficiently high.

\footnotetext{
${ }^{25}$ In both cases, we choose an equilibrium with highest sum of agents' actions.
} 
These results are the first to reveal the significance of the lowest eigenvalue of a network graph. It is not a common measure in sociology or physics, for example, and there is only a small amount of mathematical work recently on the relationship between the link structure and the lowest eigenvalue. ${ }^{26}$ Below we describe and formally elaborate this relationship. To develop an intuition, note that $\lambda_{\min }(\mathbf{G})<0$; hence when it is smaller, it corresponds to a larger number in absolute value. When $\lambda_{\min }(\mathbf{G})$ is small, the graph contains many links that connect distinct sets of agents - actions then have greater impact on others' play, and we say the graph has low absorptive capacity. Thus, large $\lambda_{\min }(\mathbf{G})$ is key to stable outcomes.

\section{A. Tool: Potential Function}

We begin by using the theory of potential games to reformulate the equilibrium conditions. Consider a game where player $i$ chooses $x_{i} \in X_{i}, X=\prod_{i} X_{i}$, and payoffs are $V_{i}\left(x_{i}, \mathbf{x}_{-i}\right)$. From Monderer \& Shapley (1996), a function $\varphi\left(x_{i}, \mathbf{x}_{-i}\right)$ is a potential function for this game if and only if for all $i$

$$
\varphi\left(x_{i}, \mathbf{x}_{-i}\right)-\varphi\left(x_{i}^{\prime}, \mathbf{x}_{-i}\right)=V_{i}\left(x_{i}, \mathbf{x}_{-i}\right)-V_{i}\left(x_{i}^{\prime}, \mathbf{x}_{-i}\right) \text { for all } x_{i}, x_{i}^{\prime} \in X_{i} \text { and all } \mathbf{x}_{-i} \in X_{-i}
$$

For $x_{i} \in \mathbb{R}$ and continuous, twice-differentiable payoffs $V_{i}$, there exists a potential function if and only if $\frac{\partial^{2} V_{i}(\mathbf{x})}{\partial x_{i} \partial x_{j}}=\frac{\partial^{2} V_{i}(\mathbf{x})}{\partial x_{j} \partial x_{i}}$ for all $i \neq j$.

Examining the game with quadratic payoffs $\widetilde{U}_{i}$, it has a potential function since $\frac{\partial^{2} \widetilde{U}_{i}}{\partial x_{i} \partial x_{j}}=$ $-\delta g_{i j}=\frac{\partial^{2} \widetilde{U}_{i}}{\partial x_{j} \partial x_{i}}$. The following function

$$
\varphi(\mathbf{x} ; \delta, \mathbf{G})=\sum_{i=1}^{n}\left(x_{i}-\frac{1}{2} x_{i}^{2}\right)-\frac{1}{2} \delta \sum_{i=1}^{n} \sum_{j=1}^{n} g_{i j} x_{i} x_{j}
$$

is a potential function for $\widetilde{U}_{i} ; \frac{\partial \widetilde{U}_{i}}{\partial x_{i}}=\frac{\partial \varphi}{\partial x_{i}}$. Notice that $\varphi(\mathbf{x} ; \delta, \mathbf{G})$ is strictly concave in each $x_{i}$.

We can use this potential function to find the equilibria for all games in our class, since all the games have the same equilibria as the game with payoffs $\widetilde{U}_{i} \cdot{ }^{27}$ Consider the following constrained optimization problem $(\mathrm{P})$ :

\footnotetext{
${ }^{26}$ See Desai \& Rao (1994), Alon \& Sudakov (2000), Ye, Fan \& Liang (2008), Bell, Cvetković, Rowlinson \& Simić (2008a, 2008b), Trevisan (2009).

${ }^{27}$ In the terminology of Morris \& Ui (2004), the games in our class are "best-response potential games," since they are "best-response equivalent" and one of the games is a potential game.
} 


$$
\max _{\mathbf{x}} \varphi(\mathbf{x} ; \delta, \mathbf{G}) \quad \text { s.t. } \quad \forall i, x_{i} \geq 0
$$

and consider the Kuhn-Tucker conditions, such as for $x_{i}$

$$
\frac{\partial \varphi}{\partial x_{i}}=0 \text { and } x_{i}>0 ; \text { or } \frac{\partial \varphi}{\partial x_{i}} \leq 0 \text { and } x_{i}=0
$$

The Kuhn-Tucker condition for each $x_{i}$ exactly correspond to an agent $i$ 's individual best response (2). ${ }^{28}$ Thus we have:

Lemma 1. A profile $\mathbf{x}$ is a Nash equilibrium of any game with the best reply function $f_{i}(\mathbf{x})=$ $\max \left(0,1-\delta \sum_{j=1}^{n} g_{i j} x_{j}\right)$ if and only if $\mathbf{x}$ satisfies the Kuhn-Tucker conditions of problem $(P)$.

The set of Nash equilibria for a given $\mathbf{G}$ and $\delta$ is then equal to the local and global maxima and saddle points of the potential function $\varphi(\mathbf{x} ; \delta, \mathbf{G})$ on $\mathbb{R}_{+}^{n}$.

Solving for the Nash equilibria for these games is then a quadratic programming problem. To see this clearly, rewrite the potential function as $\varphi(\mathbf{x} ; \delta, \mathbf{G})=\mathbf{x}^{T} \mathbf{1}-\frac{1}{2} \mathbf{x}^{T}(\mathbf{I}+\delta \mathbf{G}) \mathbf{x}$. Quadratic optimization has been well-studied in mathematics and computer science (e.g. Lee, Tam \& Yen (2005)). The heart of the problem is the matrix $\mathbf{I}+\delta \mathbf{G}$. When $\mathbf{I}+\delta \mathbf{G}$ is positive definite, $\varphi(\mathbf{x} ; \delta, \mathbf{G})$ is concave, there is a unique solution to $(\mathrm{P})$, and $(\mathrm{P})$ is solvable in polynomial time. In general, however, solving the problem (P) is NP-complete (Pardalos \& Vavasis (1991)) and finding all the maxima and saddle points requires exponential time. ${ }^{29}$ Yet knowledge about this problem gives us enormous tractability to characterize equilibria, as we discuss next.

\section{B. Lowest Eigenvalue of Graph and Nash Equilibria}

\section{B.1. Unique Equilibria: Convex Optimization $-\lambda_{\min }(\mathbf{G})$ is large}

We first obtain a strong, intuitive condition for the uniqueness of equilibria which is the strongest in the literature. A traditional uniqueness condition derives from contraction of the best reply function, which occurs if and only if $\delta<1 / \rho(\mathbf{G})$ (see Appendix). Thus, from standard arguments

\footnotetext{
${ }^{28} \varphi\left(x_{i}, \mathbf{x}_{-i}\right)$ is strictly concave in each $x_{i}$, so for any $\mathbf{x}_{-i}$ a single $x_{i}$ satisfies the $i^{t h}$ Kuhn-Tucker condition.

${ }^{29}$ While computer scientists have developed many algorithms that would quickly identify one equilibrium, only enumerative methods are guaranteed to find them all (Murty \& Yu (1988)).
} 
a unique equilibrium exists if $\delta<1 / \rho(\mathbf{G}) \cdot{ }^{30}$ Our next result shows that this sufficient condition is not necessary. Our condition relies on $\lambda_{\min }(\mathbf{G})$ and holds even when the best replies are not contracting.

The major insight is that individual responses to others' actions are geometrically structured by the potential function. The whole action profile moves along the surface of the potential. It is the curvature of the potential function, and the network which gives the potential its shape which determines the number and character of equilibrium outcomes.

We find that the lowest eigenvalue of a graph, $\lambda_{\min }(\mathbf{G})$, captures the relevant shape of the potential. The potential function $\varphi(\mathbf{x} ; \delta, \mathbf{G})$ is strictly concave if and only if $\delta<-1 / \lambda_{\min }(\mathbf{G})$. This follows from the Hessian of the potential which is equal to $\nabla^{2} \varphi=-(\mathbf{I}+\delta \mathbf{G})$, and $\mathbf{I}+\delta \mathbf{G}$ is positive definite if and only if $\delta<-1 / \lambda_{\min }(\mathbf{G})$. We can then invoke the theory of convex optimization (as applied to quadratic programming) to analyze problem $(\mathrm{P})$. If $\varphi(\mathbf{x} ; \delta, \mathbf{G})$ is strictly concave, there is a unique global maximum, for which the Kuhn-Tucker conditions are necessary and sufficient. ${ }^{31}$ Thus, we have

Proposition 2. If $\delta<-1 / \lambda_{\min }(\mathbf{G})$, there is a unique Nash equilibrium.

This condition is often both necessary and sufficient for a unique equilibrium. ${ }^{32}$ For example, it is necessary and sufficient for an important class of graphs, regular graphs (we show this below).

We can also see necessity and sufficiency in particular graphs, such as the line with four players in Example 2, where $\lambda_{\min }(\mathbf{G})=-\frac{\sqrt{5}-1}{2}$.

Proposition 2 provides the best known sufficient condition for unique equilibria in these games. It is stronger than the two previous conditions: Ballester \& Calvó-Armengol (2007, Proposition 1) show that there exists a unique equilibrium if $\delta<1 / \rho(\mathbf{G})$, which corresponds to the condition for contracting best replies. For any $\mathbf{G},-1 / \lambda_{\min }(\mathbf{G}) \geq 1 / \rho(\mathbf{G})$, and this inequality is strict when no component of $\mathbf{G}$ is bipartite. ${ }^{33}$ Ballester, Calvó-Armengol \& Zenou (2006, Theorem 1) show there exists a unique interior equilibrium for $\delta<1 /\left(1+\lambda_{\max }(\mathbf{C}-\mathbf{G})\right)$. For any $\mathbf{G},-1 / \lambda_{\min }(\mathbf{G}) \geq$

\footnotetext{
${ }^{30}$ Ballester \& Calvo-Armengol $\left.(2007)\right)$ also show there is a unique equilibrium if $\delta<1 / \rho(\mathbf{G})$ by using the mathematics of linear-complementarity problems.

${ }^{31}$ The result is a network case of Neyman's (1997) finding for general potential games with convex compact action spaces that shows there is a unique correlated equilibrium when the potential is strictly concave. Bergemann \& Morris (2007) generalize this finding to games of incomplete information.

${ }^{32}$ In addition, we know that the unique equilibrium varies continuously with $\delta$ when $\delta<-1 / \lambda_{\min }(\mathbf{G})$.

${ }^{33}$ See Theorem 0.13 in Cvetković et al. (1979).
} 
$1 /\left(1+\lambda_{\max }(\mathbf{C}-\mathbf{G})\right)$, and this inequality is strict, for example, when $k_{\max }(\mathbf{G})<n / 2$. (See Appendix for details.)

\section{B.2. Multiple Equilibria and Corner Outcomes: Non-Convex Optimization $-\lambda_{\min }(\mathbf{G})$ is small}

We next tackle equilibria when $\delta>-1 / \lambda_{\min }(\mathbf{G})$. The potential $\varphi(\mathbf{x} ; \delta, \mathbf{G})$ is not concave; ${ }^{34}$ problem (P) falls in the domain of non-convex optimization, and multiple equilibria are possible.

Yet, multiplicity does not mean that "anything goes." We show that for $\delta>-1 / \lambda_{\min }(\mathbf{G})$ there always exists an equilibrium that is a corner. In the following section, we show that corner equilibria are the only stable equilibria in this range.

We use three facts. First, for any $\delta$ and $\mathbf{G}$, there exists at least one vector, denoted $\mathbf{x}^{*}(\delta, \mathbf{G})$, that globally maximizes the potential $\varphi(\mathbf{x} ; \delta, \mathbf{G}){ }^{35}$ Second, by Lemma 1 any maximum of the potential is a Nash equilibrium, so $\mathbf{x}^{*}(\delta, \mathbf{G})$ is a Nash equilibrium. Third, if $\delta>-1 / \lambda_{\min }(\mathbf{G})$ the potential is a non-concave quadratic function. Then, there must be a direction along which the potential increases without bound. Hence, the vector $\mathbf{x}^{*}(\delta, \mathbf{G})$ must not be interior, and for this equilibrium $A \neq N$. These facts give us:

Proposition 3. If $\delta>-1 / \lambda_{\min }(\mathbf{G})$, there exists an equilibrium which is a corner.

Intuitively, when $\delta$ is high relative to the absorptive capacity of the graph, individual responses are amplified, not dampened, by the graph. Under strategic substitutes, as an individual's action increases, his neighbors' actions decrease. If the graph cannot absorb the payoff impact and reactions rebound, some boundaries of the action space must be reached. This feature depends on how links connect agents in the graph, which we will describe at length in section $\mathrm{V}$ below.

The following example illustrates Propositions 2 and 3.

Example 4. Unique and Corner Equilibria. Consider the graph in Figure 4. $\lambda_{\min }(\mathbf{G})=1 / 3$. For $\delta<1 / 3$, there is a unique equilibrium, where each agent plays $\frac{1}{1+3 \delta}$. For $1 / 3<\delta \leq 1$ there

\footnotetext{
${ }^{34}$ When $\delta=-1 / \lambda_{\min }(\mathbf{G})$, the potential is concave but not strictly concave. While multiple equilibria may emerge, the equilibrium set is strongly structured. Equilibria form a convex set, and all equilibria yield the same aggregate action. In addition, one equilibrium is the limit of the unique equilibrium for $\delta<-1 / \lambda_{\min }(\mathbf{G})$ as $\delta$ tends to $-1 / \lambda_{\min }(\mathbf{G})$ from below.

${ }^{35}$ Problem $(\mathrm{P})$ is equivalent to maximizing $\varphi(\mathbf{x})$ over $[0,1]^{n}$, hence a maximum exists because the domain is compact and $\varphi(\mathbf{x})$ is continuous.
} 

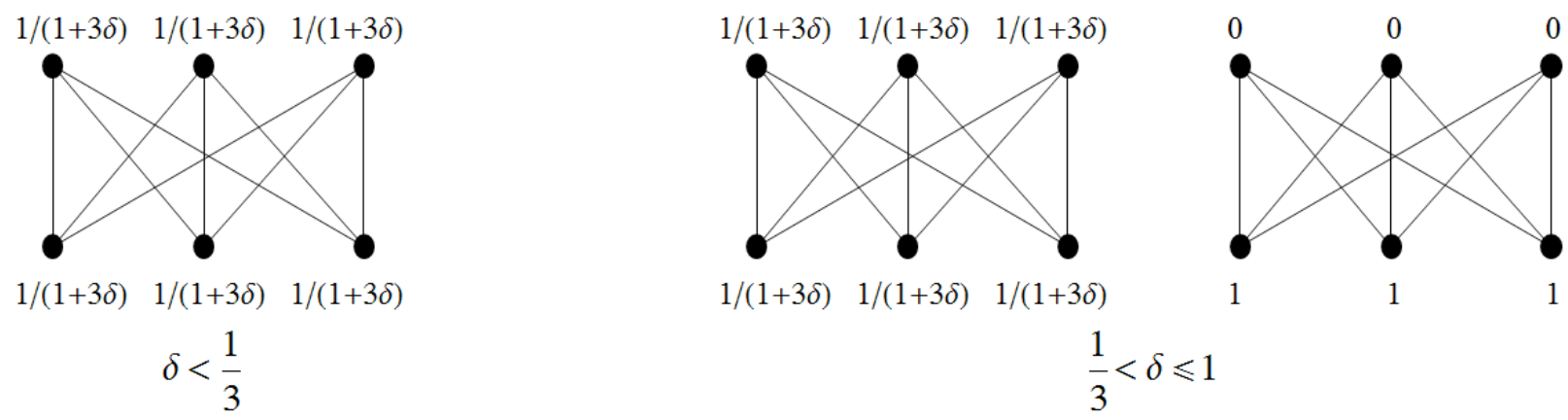

Figure 4: Lowest Eigenvalue and Unique and Corner Equilibria

are three equilibria: the interior equilibrium where each agent plays $\frac{1}{1+\delta 3}$, and two specialized equilibria where agents on one side play 1 and agents on the other side play 0. In this range, the interior equilibrium is a saddle point and the specialized equilibria are global maxima of the potential.

This example generalizes; Propositions 2 and 3 give tight conditions for particular graphs and for all regular graphs. When every agent has $k$ neighbors, for any $\delta \in[0,1]$ it is an equilibrium for each agent to play $x_{i}=1 /(1+\delta k)$. If $\delta<-1 / \lambda_{\min }(\mathbf{G})$, this is the unique equilibrium. If $\delta>-1 / \lambda_{\min }(\mathbf{G})$, an interior vector cannot maximize the potential. Hence there must be another equilibrium, which is a corner. ${ }^{36}$

Corollary 1. For regular graphs, there is a unique equilibrium if and only if $\delta<-1 / \lambda_{\min }(\mathbf{G})$ and this equilibrium is interior. If $\delta \geq-1 / \lambda_{\min }(\mathbf{G})$ there are both interior and corner equilibria.

Combining Propositions 2, 3, and previous results, for a graph $\mathbf{G}$ we can divide the range of $\delta$ into four parts. In the lowest part, $0 \leq \delta<\underline{\delta}_{B C A Z}$ there is a unique and interior equilibrium. The equilibrium vector is $\mathbf{x}=(\mathbf{I}+\delta \mathbf{G})^{-1} \mathbf{1}=\mathbf{1}-\delta \mathbf{c}(-\delta, \mathbf{G})$. In the lower-middle range, $\underline{\delta}_{B C A Z} \leq \delta<$ $-1 / \lambda_{\min }(\mathbf{G})$, there is a unique equilibrium, which is either interior or corner. In the upper-middle range, $-1 / \lambda_{\min }(\mathbf{G}) \leq \delta<1$, multiple equilibria are possible, and a corner equilibrium always exists. For active agents, in any equilibrium, $\mathbf{x}_{A}=\left(\mathbf{I}+\delta \mathbf{G}_{A}\right)^{-1} \mathbf{1}=\mathbf{1}-\delta \mathbf{c}\left(-\delta, \mathbf{G}_{A}\right)$. At $\delta=1$, for any graph there is an equilibrium vector where $\mathbf{x}_{A}=\mathbf{1}$, and the active agents constitute a maximal independent set of the graph. We see these ranges in Examples 1, 2, 3, and 4.

\footnotetext{
${ }^{36}$ At $\delta=-1 / \lambda_{\min }(\mathbf{G})$, there are multiple equilibria (interior and corner), and they each maximize the potential.
} 


\section{Stable Equilibria}

In this section we delve more deeply into the problem of multiple equilibria and identify those equilibria which are stable. We show the lowest eigenvalue of a graph is key to stability, as it is key to unique equilibria.

\section{A. Asymptotically Stable Equilibria}

We consider a continuous adjustment process, asking when a Nash equilibrium $\mathbf{x}$ is an asymptotically stable stationary state (Weibull (1995)) of the following system of differential equations:

$$
\begin{gathered}
\dot{x}_{1}=h_{1}(\mathbf{x})=f_{1}(\mathbf{x} ; \delta, \mathbf{G})-x_{1} \\
\vdots \\
\dot{x}_{n}=h_{n}(\mathbf{x})=f_{n}(\mathbf{x} ; \delta, \mathbf{G})-x_{n}
\end{gathered}
$$

where $f_{i}(\mathbf{x} ; \delta, \mathbf{G})$ is agent $i$ 's best response (2). In vector notation $\dot{\mathbf{x}}=H(\mathbf{x})$, where $H=$ $\left(h_{1}, \ldots h_{n}\right): \mathbb{R}^{n} \rightarrow \mathbb{R}^{n}$. By construction, $\mathbf{x}$ is a stationary state of this system if and only if $\mathbf{x}$ is a Nash equilibrium of our games. ${ }^{37}$ A Nash equilibrium $\mathbf{x}$ is asymptotically stable when this system of differential equations converges back to $\mathbf{x}$ following any small enough perturbation. ${ }^{38}$

Next, say that a (local or global) maximum $\mathbf{x}$ of the potential is locally strict if there is an open neighborhood of $\mathbf{x}$ in $\mathbb{R}_{+}^{n}$ on which $\varphi$ does not have any other maximum. We can then show there is a one-to-one relationship between locally strict maxima and stable equilibria:

Lemma 2. An equilibrium $\mathbf{x}$ is asymptotically stable if and only if $\mathbf{x}$ is a locally strict maximum of the potential.

To prove this result note that the opposite of the potential function, $-\varphi$, provides a natural Lyapunov function for the system of differential equations. In particular, the potential must

\footnotetext{
${ }^{37}$ We also looked at discrete Nash tâtonnement, as in Bramoullé \& Kranton (2007). We find equilibria stable to Nash tâtonnement are (almost always) asymptotically stable. But the reverse is not true, and Nash tâtonnement stable equilibria may fail to exist in large regions of the parameter space. (Proofs are available upon request.) In contrast, asymptotically stable equilibria exist generically, as shown below.

${ }^{38}$ Following Weibull (1995, Definition 6.5, p.243), we define asymptotic stability as follows: Introduce $B(\mathbf{x}, \varepsilon)=$ $\left\{\mathbf{y} \in \mathbb{R}_{+}^{n}:\|\mathbf{y}-\mathbf{x}\|<\varepsilon\right\}$ and $\boldsymbol{\xi}(t, \mathbf{y})$ the value at time $t$ of the unique solution to the system differential equations that starts at $\mathbf{y}$. (So, $\boldsymbol{\xi}(0, \mathbf{y})=\mathbf{y})$. By definition, $\mathbf{x}$ is Lyapunov stable if $\forall \varepsilon>0, \exists \eta>0: \forall \mathbf{y} \in B(\mathbf{x}, \eta)$, $\forall t \geq 0, \boldsymbol{\xi}(t, \mathbf{y}) \in B(\mathbf{x}, \varepsilon)$. Then, $\mathbf{x}$ is asymptotically stable if it is Lyapunov stable and if $\exists \varepsilon>0: \forall \mathbf{y} \in B(\mathbf{x}, \varepsilon)$, $\lim _{t \rightarrow \infty} \boldsymbol{\xi}(t, \mathbf{y})=\mathbf{x}$.
} 
always increase along the trajectories of the system: $\frac{d}{d t} \varphi(\mathbf{x}(t))=\sum_{i} \frac{\partial \varphi}{\partial x_{i}} \dot{x}_{i}=\nabla \varphi(\mathbf{x}) \cdot H(\mathbf{x})>0$ if $\mathbf{x}$ is not an equilibrium.

Thus, stability eliminates the Nash equilibria that are saddle points of the potential and maxima that are not locally strict. Observe that for any $\mathbf{G}$ and almost every $\delta$, the number of equilibria is finite and, hence, maxima of the potential are all locally strict. Since for any $\delta$ and $\mathbf{G}$, the potential has a global maximum, a stable equilibrium exists for almost every $\delta$.

\section{B. Necessary and Sufficient Condition for Stablility}

Here we find a necessary and sufficient condition for stable equilibria by looking at the shape of the potential at an equilibrium $\mathbf{x}$. For unique equilibria, we looked at global concavity of the potential. Here we focus on the shape of the potential locally, in the neighborhood of an equilibrium $\mathbf{x}$. We find that for an equilibrium $\mathbf{x}$, with active agents $A, \lambda_{\min }\left(\mathbf{G}_{A}\right)$ gives (almost always) a necessary and sufficient condition for stability.

To make our argument, we establish that we need only look at active agents and the subgraph $\mathbf{G}_{A}$. For inactive agents, there are two types: (a) those for whom a small reduction in neighbors' actions would not lead them to change their play; i.e., agents for whom $\delta \sum_{j} g_{i j} x_{j}>1$; and (b) those for whom a small reduction would lead them to increase their play; i.e., $\delta \sum_{j} g_{i j} x_{j}=1$. The first type can be safely ignored (see Appendix). The second type, which we call knife-edge agents must be examined, since even a small reduction in a neighbor's action would lead them to increase their play. We show in the Appendix however, that for any graph $\mathbf{G}$ for almost every $\delta$, there are no knife-edge agents in any equilibrium. Thus, almost always, the relevant shape of the potential around an equilibrium $\mathbf{x}$ is determined only by $\mathbf{G}_{A}$. In particular, from $\mathbf{G}_{A}$ we derive the curvature of the potential on the subset of the action space restricted to active agents.

Generically: When $\lambda_{\min }\left(\mathbf{G}_{A}\right)$ is large relative to $\delta$, the potential function is strictly concave on the action space of active agents. The graph absorbs the impact of changes in play, and the equilibrium is stable. When $\lambda_{\min }\left(\mathbf{G}_{A}\right)$ is small relative to $\delta$, the potential function is not concave on the action space of action agents. A small change reverberates through the network, and the equilibrium is not stable. This gives a generic necessary and sufficient condition for stability: an equilibrium is stable if and only if $\delta<-1 / \lambda_{\min }\left(\mathbf{G}_{A}\right) \cdot{ }^{39}$

\footnotetext{
${ }^{39}$ This condition is written for non-empty graphs $\mathbf{G}_{A}$. An equilibrium $\mathbf{x}$ with active agents $A$ is stable for any
} 
Non-generically: In the presence of knife-edge agents, $\delta<-1 / \lambda_{\min }\left(\mathbf{G}_{A}\right)$ is necessary for stability. We derive the precise necessary and sufficient condition in this case in the Appendix. Summarizing:

Proposition 4. Consider a graph $\boldsymbol{G}$ and a Nash equilibrium $\mathbf{x}$ with active agents $A$. If there are no knife-edge agents, $\mathbf{x}$ is stable if and only if $\delta<-1 / \lambda_{\min }\left(\mathbf{G}_{A}\right)$. If there are knife-edge agents, if $\mathbf{x}$ is stable then $\delta<-1 / \lambda_{\min }\left(\mathbf{G}_{A}\right)$.

\section{Shape of Stable Equilibria}

We can also determine the shape of stable equilibria. When $\delta<-1 / \lambda_{\min }(\mathbf{G})$, by Proposition 2 , there is a unique equilibrium. This equilibrium is interior or corner, and it is stable. When $\delta>-1 / \lambda_{\min }(\mathbf{G})$, there can be multiple equilibria and by Proposition 3 at least one equilibrium is a corner. Since for interior equilibria $A=N$, our stablility condition eliminates all the interior equilibria in this range,

Corollary 2. For a graph $\mathbf{G}$, if $\delta>-1 / \lambda_{\min }(\mathbf{G})$, all stable equilibria are corners.

The following examples illustrate the lowest eigenvalue stability condition and the shape of stable of equilibria.
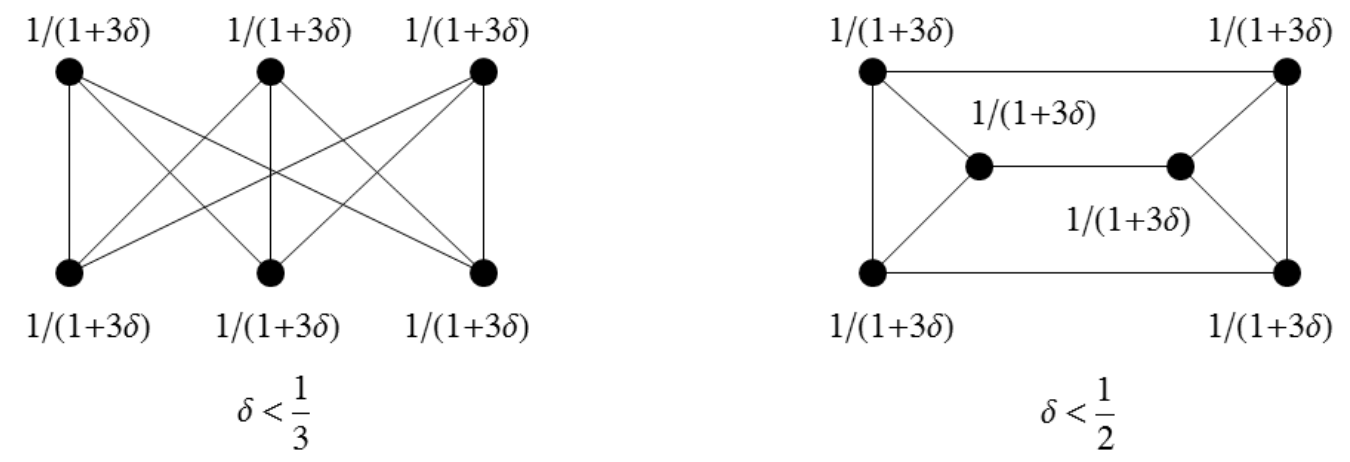

Figure 5: Lowest Eigenvalue and Stable Equilibria

$\delta$ if $A$ is such that $\mathbf{G}_{A}$ is empty and there are no knife edge agents. $A$ is then a maximal independent set, the equilibrium is specialized, and inactive agents are connected to at least two active ones. 
Example 5. Stable Equilibria and the Lowest Eigenvalue. Consider the graph on the left of Figure 5, which is complete bipartite, and the profile $x_{i}=\frac{1}{1+3 \delta}$ for all $i$ which is a Nash equilibrium for any $\delta \in[0,1]$. For this graph, $\lambda_{\min }(\mathbf{G})=-3$, hence this equilibrum is stable for $\delta<1 / 3$. For $1 / 3<\delta$, only corner equilibria are stable. Next consider the "prism" graph in Figure 5. Since it is also regular with $k=3, x_{i}=\frac{1}{1+3 \delta}$ is a Nash equilibrium for any $\delta \in[0,1]$. Here, $\lambda_{\min }(\mathbf{G})=-2$, and hence, the interior equilibrium is stable for $\delta<1 / 2$. For $1 / 2<\delta$, only corner equilibria are stable. For intuition, compare what happens after perturbing one agent's play in the bipartite graph to perturbing one agent's play in the prism graph. Let some agent $i$ play $\frac{1}{1+3 \delta}+\varepsilon$ In the bipartite graph, all three agents on the other side will adjust their play by the full amount. In the prism graph, two of the agents linked to $i$ are also linked to each other and "share" the adjustment. Hence, the interior equilibrium is stable for a higher $\delta$ in the prism graph than in the complete bipartite graph.

Example 6. Stable Equilibria versus Nash Equilibria. The stability condition can eliminate many Nash equilibria in the range $\delta>-1 / \lambda_{\min }(\mathbf{G})$. For example, in the complex network pictured in Figure 3, at $\delta=0.35$ two among the three equilibria are stable. At $\delta=0.55$, only seven among the thirty-one equilibria are stable.

This analysis gives a general intution that stable equilibria involve smaller sets of active agents. Interior equilibria - where everyone takes positive action - are only stable when they are the unique equilibrium. Otherwise, only corner equilibria are stable. Furthermore, the sets of active agents of stable equilibria are minimal in terms of inclusion. Consider two equilibria $\mathbf{x}$ and $\mathbf{x}^{\prime}$ with active agents $A$ and $A^{\prime}$. If $A \subset A^{\prime}$, then stability of $\mathbf{x}$ implies instability of $\mathbf{x}^{\prime}$ (see Appendix). This is consistent with the fact that $A \subset A^{\prime} \Rightarrow \lambda_{\min }\left(\mathbf{G}_{A}\right) \geq \lambda_{\min }\left(\mathbf{G}_{A^{\prime}}\right) .{ }^{40}$ Smaller subgraphs have more absorptive capacity.

To summarize our stability results: For any graph $\mathbf{G}$ and almost every $\delta$, a stable equilibrium exists. If $\delta<-1 / \lambda_{\min }(\mathbf{G})$, the unique equilibrium is asymptotically stable; it is a corner or an interior equilibrium. If $\delta>-1 / \lambda_{\min }(\mathbf{G})$, the stable equilibria are corners. The stability conditions focus our attention on particular equilibria with small sets of active agents, and Section VI conducts comparative statics on these equilibria.

\footnotetext{
${ }^{40}$ This relationship follows from the interlacing eigenvalue theorem, e.g. Horn \& Johnson (1985, p.185). Note that any perturbation on the smaller subgraph can be replicated on the larger one, but not vice versa.
} 


\section{Lowest Eigenvalue of a Graph}

The previous sections show the lowest eigenvalue of a graph is critical to unique and stable equilibria. Here we how this graph statistic relates to network structure.

We can gain much intuition from looking at the two networks in Figure 5. For the complete bipartite graph $\lambda_{\min }(\mathbf{G})=-3$, and for the prism graph $\lambda_{\min }(\mathbf{G})=-2$. Each has nine links, and each is a regular graph. But in the complete bipartite graph there are two distinct sets of agents, with links between the sets but not within each set. The complete bipartite graph has no triangles. In the prism graph, on the other hand, there are triangles - what sociologists call "triadic closures;" friends of an agent $i$ are also friends with each other. Loosely speaking, the lowest eigenvalue involves a tradeoff between more links and more closure.

We can formalize this intuition as follows. Let $\mathcal{G}$ be the set of all possible graphs for $n$ agents. Let $\mathbf{G}_{*} \in \mathcal{G}$ be a graph with the smallest lowest eigenvalue; i.e., $\lambda_{\min }\left(\mathbf{G}_{*}\right) \leq \lambda_{\min }(\mathbf{G})$ for all $\mathbf{G} \in \mathcal{G}$.

First, $\mathbf{G}_{*}$ is a complete bipartite graph with as equal size sides as possible. ${ }^{41}$

Eigenvalue Result 1. $\mathbf{G}_{*}$ is a complete bipartite graph. For $n$ even, $k=n / 2$ for all agents. For $n$ odd, $\frac{n+1}{2}$ agents have $\frac{n-1}{2}$ links, and $\frac{n-1}{2}$ agents have $\frac{n+1}{2}$ links.

Intuitively, the complete bipartite structure maximizes the amplification of shocks. A change in play by any agent reverberates through the network. In contrast, a complete graph, where all agents are connected (so there is complete triadic closure), contains the shocks by any agent. ${ }^{42}$

Second, for any graph $\mathbf{G} \neq \mathbf{G}_{*}$ we can cut and add links to $\mathbf{G}$ to yield a $\mathbf{G}^{\prime}$ that has a smaller lowest eigenvalue. We essentially make the graph "more bipartite." For any G, there exists a partition of the population in two sets $R$ and $S$ such that adding links between or cutting links within the sets weakly reduces the lowest eigenvalue. We identify these sets from an eigenvector associated with $\lambda_{\min }(\mathbf{G}) .^{43}$

Eigenvalue Result 2. Consider a graph $\mathbf{G} \neq \mathbf{G}_{*}$. Let $\mathbf{u}$ be an eigenvector for $\lambda_{\min }(\mathbf{G})$, and let $R=\left\{i: u_{i} \geq 0\right\}$ and $S=\left\{i: u_{i}<0\right\}$. Form a new graph $\mathbf{G}^{\prime}$ by removing any number of links $(i, j) \in R \times R$ or $S \times S$, and adding any number of links $(k, l) \in R \times S$. Then $\lambda_{\min }\left(\mathbf{G}^{\prime}\right) \leq \lambda_{\min }(\mathbf{G})$

\footnotetext{
${ }^{41}$ We thank Noga Alon for his help and providing us a proof of this result. See also Constantine (1985).

${ }^{42}$ The complete graph is the connected graph with the largest smallest eigenvalue; $\lambda_{\min }(\mathbf{C})=-1$.

${ }^{43}$ These eigenvectors give directions of the maximal perturbation of the potential.
} 
Intuitively, by adding links between sets, agents' actions directly impact more agents. Cutting links within sets reduces triadic closures, and agents' actions are less contained by the graph.

Third, a graph has the smallest lowest eigenvalue when it has as many links as possible with no triangles. More links increase the impact of shocks, but triadic closures contain them. We have: ${ }^{44}$

Eigenvalue Result 3. $\mathbf{G}_{*}$ is equivalent to the graph $\mathbf{G} \in \mathcal{G}$ with the greatest number of links and no triadic closure.

The problem of finding the graph for $n$ agents with the smallest lowest eigenvalue for a fixed number of links is much more difficult and has only recently been addressed by mathematicians. ${ }^{45}$

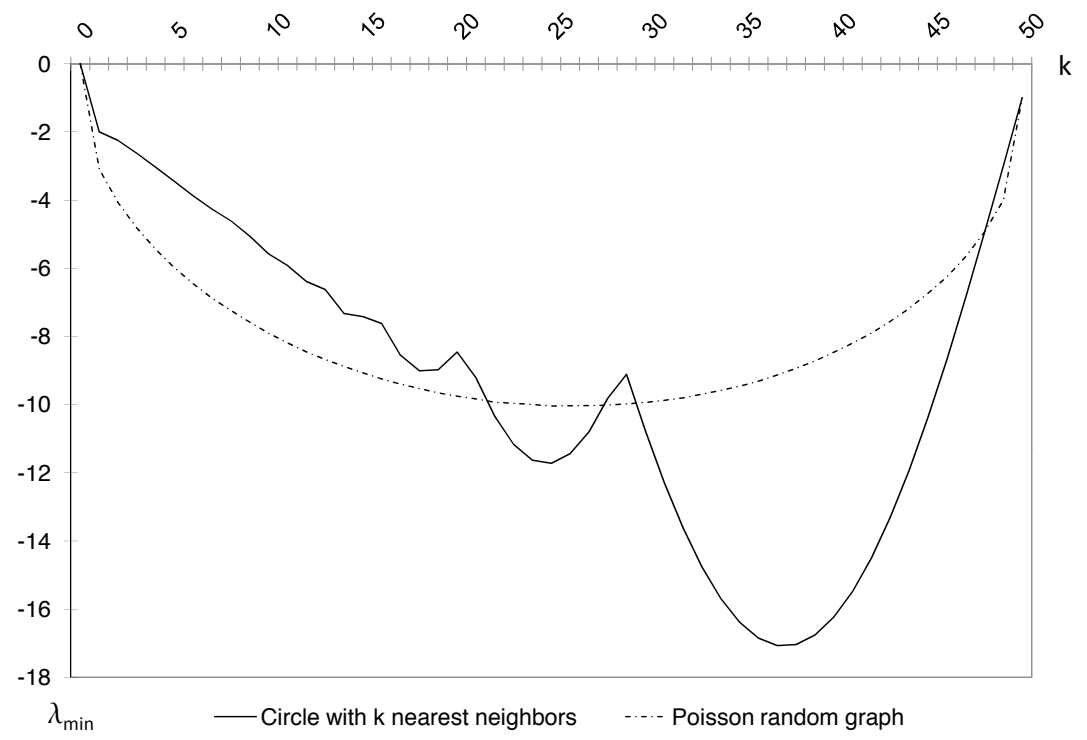

Figure 6: Lowest Eigenvalue and Network Links

For particular gaph families, we can compute lowest eigenvalues to see the tradeoffs as links are added to a network. We depict in Figure 6 how the lowest eigenvalue varies with number of links for two well-known structures: Bernouilli random graphs and circles. In Bernouilli random graphs, the expected lowest eigenvalue is first decreasing and then increasing in the probability

\footnotetext{
${ }^{44}$ See Theorem 2, p.6, and Exercise 4, p.28, in Bollobas (1998).

${ }^{45}$ See Bell, Cvetković, Rowlinson \& Simić (2008a, 2008b).
} 
of connection. Starting from the empty graph, most links are between sets of agents. The new links, however, eventually form more triangles (i.e., links within sets of agents), and the absorptive capacity of the network increases. This relationship is more irregular for the circle, despite the regularity of its structure. The lowest eigenvalue for the circle also reaches its minimum for a higher number of connections than the Bernoulli graph. ${ }^{46}$

\section{Comparing Equilibria}

In this section we compare equilibrium outcomes in terms of aggregate play, $\sum_{i=1}^{n} x_{i}$. We can use the potential function to do so, since the potential evaluated at an equilibrium is always equal to one-half of total play; that is, for a given $\delta$ and $\mathbf{G}$ and an equilibrium $\mathbf{x}(\delta, \mathbf{G}), \varphi(\mathbf{x} ; \delta, \mathbf{G})=$ $\frac{1}{2} \sum_{i=1}^{n} x_{i}(\delta, \mathbf{G}) \cdot{ }^{47}$

\section{A. Aggregate Play for a given $\delta$ and $\mathbf{G}$}

Equilibria with fewer active agents - in terms of set of inclusion - always have a higher level of aggregate action. That is, consider an equilibrium $\mathbf{x}$ with active agents $A$ and equilibrium $\mathbf{x}^{\prime}$ with active agents $A^{\prime}$, where $A \subset A^{\prime}$. Then, $\sum_{i} x_{i}^{\prime} \leq \sum_{i} x_{i}$. Concentrating actions on fewer agents increases overall play. This finding reinforces our intuition about small sets of active agents. Fewer active agents is related to both higher aggregate play and greater stability.

\section{B. Comparative Statics-Aggregate Play for different $\delta$ and G}

Next we conduct comparative statics on $\delta$ and $\mathbf{G}$. Comparative statics are difficult in games of strategic substitutes since direct and indirect effects generally pull in opposite directions. Consider a change in a parameter that induces agent $i$ to increase her action. In response, $i$ 's neighbors decrease their actions, and their neighbors increase their actions, and so on. The resulting impact on play could be complicated and nonmonotonic. We use the potential function to overcome these difficulties, and we obtain clean local and global comparative static results.

\footnotetext{
${ }^{46}$ We also studied small-world graphs. Consider the original Watts \& Strogatz (1998) procedure: From a circle, rewire each link at random with probability $p$. As $p$ varies from 0 to 1 , the expected lowest eigenvalue varies smoothly and monotonically from the lowest eigenvalue of the circle to the expected lowest eigenvalue of the Bernouilli random graph.

${ }^{47}$ Let $\mathbf{x}$ be an equilibrium with active agents $A$. Since $\mathbf{x}_{N-A}=\mathbf{o}, \mathbf{x}^{T}(\mathbf{I}+\delta \mathbf{G}) \mathbf{x}=\mathbf{x}_{A}^{T}\left(\mathbf{I}+\delta \mathbf{G}_{A}\right) \mathbf{x}_{A}$. By Proposition $1,\left(\mathbf{I}+\delta \mathbf{G}_{A}\right) \mathbf{x}_{A}=\mathbf{1}$. Since $\mathbf{x}_{A}^{T} \mathbf{1}=\mathbf{x}^{T} \mathbf{1}, \varphi(\mathbf{x})=\frac{1}{2} \mathbf{x}^{T} \mathbf{1}$.
} 


\section{B.1. Global Comparative Statics-Higher $\delta$, More Links}

Consider a $\delta$ and $\mathbf{G}$ and an equilibrium $\mathbf{x}^{*}(\delta, \mathbf{G})$ which is a global maximum of $\varphi(\mathbf{x} ; \delta, \mathbf{G})$. This equilibrium has the highest aggregate play of all equilibria for $\delta$ and $\mathbf{G}$. We show that any large or small increase in $\delta$ or any additional link to the graph always leads to an equilibrium with lower total play. Thus, while some agents may increase their actions, the decreases dominate.

Proposition 5. Consider a $\delta$ and $\mathbf{G}$ and an equilibrium $\mathbf{x}^{*}(\delta, \mathbf{G})$. Consider a $\delta^{\prime}$ and $\mathbf{G}^{\prime}$ where $\delta^{\prime} \geq \delta$ and $\mathbf{G}$ is a subgraph of $\mathbf{G}^{\prime}$ and any equilibrium vector $\mathbf{x}\left(\delta^{\prime}, \mathbf{G}^{\prime}\right)$. Then

$$
\sum_{i=1}^{n} x_{i}\left(\delta^{\prime}, \mathbf{G}^{\prime}\right) \leq \sum_{i=1}^{n} x_{i}^{*}(\delta, \mathbf{G})
$$

To understand this result, notice that for any vector $\mathbf{x}, \varphi\left(\mathbf{x} ; \delta^{\prime}, \mathbf{G}^{\prime}\right) \leq \varphi(\mathbf{x} ; \delta, \mathbf{G})$; that is, holding play fixed, the value of the potential is lower when the payoff impact is higher and/or more agents are connected. Therefore, we have

$$
\varphi\left(\mathbf{x}\left(\delta^{\prime}, \mathbf{G}^{\prime}\right) ; \delta^{\prime}, \mathbf{G}^{\prime}\right) \leq \varphi\left(\mathbf{x}^{*}\left(\delta^{\prime}, \mathbf{G}^{\prime}\right) ; \delta^{\prime}, \mathbf{G}^{\prime}\right) \leq \varphi\left(\mathbf{x}^{*}\left(\delta^{\prime}, \mathbf{G}^{\prime}\right) ; \delta, \mathbf{G}\right) \leq \varphi\left(\mathbf{x}^{*}(\delta, \mathbf{G}) ; \delta, \mathbf{G}\right)
$$

where the third inequality holds because $\mathbf{x}^{*}(\delta, \mathbf{G})$ is a global maximum of the potential $\varphi(\mathbf{x} ; \delta, \mathbf{G}){ }^{48}$ The decrease in aggregate actions is usually strict. ${ }^{49}$

Previous comparative statics results in the literature are special cases. For low $\delta$, Theorem 2 in Ballester, Calvó-Armengol \& Zenou (2006) says if $\mathbf{G}$ is a subgraph of $\mathbf{G}^{\prime}, \delta \leq \delta^{\prime}$, and there is a unique interior equilibrium $\mathbf{x}$ for $(\delta, \mathbf{G})$ and $\mathbf{x}^{\prime}$ for $\left(\delta^{\prime}, \mathbf{G}^{\prime}\right)$, then $\sum_{i} x_{i}^{\prime} \leq \sum_{i} x_{i}$. For $\delta=1$, Bramoullé \& Kranton's (2007) example for the circle generalizes to the following: a specialized equilibrium with a largest maximal independent set of agents is always a global maximum of the potential and yields highest aggregate play (see Appendix). This finding, along with Proposition 5, implies that the number of nodes in a largest maximal independent set decreases when more links are added to a graph. Galeotti, Goyal, Jackson, Vega-Redondo and Yariv (2006) noted this fact.

\footnotetext{
${ }^{48}$ In contrast, we can build examples where the lowest total effort in equilibrium is non-monotonic in $\delta$ or $\mathbf{G}$.

${ }^{49}$ See the Appendix. Precisely, suppose that $\delta<\delta^{\prime}$ and $\mathbf{G}=\mathbf{G}^{\prime}$. Then, aggregate actions decrease strictly when no global maximum on $\delta$ and $\mathbf{G}$ is specialized. In contrast, if $\mathbf{G} \varsubsetneqq \mathbf{G}^{\prime}$ and $\delta$ is unchanged, aggregate effort decreases strictly as soon as for any $\mathbf{x}^{*}(\delta, \mathbf{G})$, the new links connect active agents.
} 


\section{B.2. Local Comparative Statics-Higher $\delta$, More Links, Same Support}

We now consider local changes. We start with a stable equilibrium. We then increase the payoff impact $\delta$ or add a link to the graph and look at equilibria that have the same or smaller set of active agents as the initial equilibrium. We again find that aggregate actions decrease.

Proposition 6. For $\delta$ and $\mathbf{G}$, consider a stable equilibrium $\mathbf{x}(\delta, \mathbf{G})$ with active agents $A$. Now consider for $\delta^{\prime}$ and $\mathbf{G}^{\prime}$ where $\delta^{\prime} \geq \delta$ and $\mathbf{G}$ is a subgraph of $\mathbf{G}^{\prime}$ and consider any equilibrium $\mathbf{x}\left(\delta^{\prime}, \mathbf{G}^{\prime}\right)$ with active agents $A^{\prime}$ such that $A^{\prime} \subset A$. Then, $\sum_{i=1}^{n} x_{i}^{\prime} \leq \sum_{i=1}^{n} x_{i}$.

Thus, we can compare a wide set of equilibria and find that total play drops when $\delta$ is higher or $\mathbf{G}$ has more links. The conditions of Proposition 6 hold when the new parameter values are "close enough" so that no new agents are active. The existence of such a close-by equilibrium when $\mathbf{G}=\mathbf{G}^{\prime}$, for example, is guaranteed for almost any $\delta$ if the increase in $\delta$ is small. ${ }^{50}$

Comparative statics of individual actions, on the other hand, are generally non-monotonic. We show the direct and indirect effects of a change in one player's action are perfectly aligned if and only if $\mathbf{G}$ is bipartite (see Appendix). This result comes from the classic property that a graph is bipartite if and only if it has no odd cycles. On bipartite graphs, a positive shock on $i$ 's action eventually leads to a decrease in the actions of every agent on the other side and to an increase in the actions of every agent on his side. In contrast, if $\mathbf{G}$ is not bipartite some direct and indirect effects must go in opposite directions.

\section{Many More Games}

Our analysis generalizes and applies to many more games. Recall in our basic model that $g_{i j}=$ $g_{j i} \in\{0,1\}$ and $\bar{x}_{i}=\bar{x}$ for all $i$. The payoff impact parameter is a positive constant $\delta \in[0,1]$, which combined with $g_{i j}>0$ for all $i j$ gives strategic substitutes. We show first how our results directly extend to a strategic substitutes game with weighted graphs $g_{i j}=g_{j i} \in[0,1]$ and heterogeneous autarkic play.

We then consider three substantively different settings. In the first, agents have idiosyncratic payoff impact parameters $\delta_{i}$. One example is games where agents care about the average of others'

\footnotetext{
${ }^{50}$ For almost any $\delta$, if $A$ is the active set of agents for an equilibrium for $\delta$ and $\mathbf{G}$ there exists $\varepsilon>0$ such that if $\left|\delta^{\prime}-\delta\right| \leq \varepsilon, A$ is also the active set of agents in an equilibrium for $\delta^{\prime}$ and $\mathbf{G}$.
} 
play, which are ubiquitous in economics. With quadratic payoffs, best replies are linear, and the impact parameters are heterogeneous and depend on an agent's position in the graph. Second, we consider strategic substitutes and complements. Pure complements have been analyzed in previous literature; the analysis is relatively straightforward, and our tools easily apply. For the more complicated general setting with any mix of substitutes or complements, we show how results extend. Finally, we discuss games with non-linear best replies.

Heterogeneous autarkic play and weighted graphs. Consider the model in the text with heterogeneous autarkic play $\bar{x}_{i}$ and a weighted graph $g_{i j}=g_{j i} \in[0,1]$. The best-reply is now

$$
f_{i}(\mathbf{x})=\max \left(0, \bar{x}_{i}-\delta \sum_{j=1}^{n} g_{i j} x_{j}\right)
$$

where $\bar{x}_{i}>0 \forall i$ and $\delta \in[0,1]$. Let $\overline{\mathbf{x}} \equiv\left(\bar{x}_{1}, \ldots \bar{x}_{n}\right)$. This model captures at least two wellknown games, written in a network form: (a) a linear-demand Cournot model with differentiated products where firms have different marginal costs, and (b) strategic private provision of public goods à la Bergstrom, Blume \& Varian (1986) where consumers have different incomes. ${ }^{51}$

Our equilibrium analysis directly extends. The Nash conditions in Proposition 1 hold. The potential here is $\varphi(\mathbf{x})=\mathbf{x}^{T} \overline{\mathbf{x}}-\frac{1}{2} \mathbf{x}^{T}(\mathbf{I}+\delta \mathbf{G}) \mathbf{x}$. Since the Hessian, $\nabla^{2} \varphi$, is not affected by $\overline{\mathbf{x}}$, all the results for unique and stable equilibria-Propositions 2, 3, and 4-apply as in the basic model.

Only the comparative statics results have to be modified. Now for an equilibrium $\mathbf{x}, \varphi(\mathbf{x})=$ $\frac{1}{2} \sum_{i=1}^{n} \bar{x}_{i} x_{i}$. We weigh individual actions by their thresholds and compare divergences from autarkic play. E.g., for a given $\delta$ and $\mathbf{G}$ and a global maximum equilibrium $\mathbf{x}^{*}(\delta, \mathbf{G})$, there is a decrease in weighted total actions for $\delta^{\prime} \geq \delta$ and $\mathbf{G}$ a subgraph of $\mathbf{G}^{\prime}$; i.e., $\sum_{i=1}^{n} \bar{x}_{i} x_{i}^{*}\left(\delta^{\prime}, \mathbf{G}^{\prime}\right) \leq$ $\sum_{i=1}^{n} \bar{x}_{i} x_{i}^{*}(\delta, \mathbf{G})$. Furthermore, we have clean comparative statics with respect to $\overline{\mathbf{x}} ;$ the weighted sum of actions increases weakly when individual thresholds increase.

Heterogenous payoff impacts and "linear-in-means" games. We now study games where

\footnotetext{
${ }^{51}$ To see this, let each individual have Cobb-Douglas utility and allocate income, $w_{i}$, between private good consumption, $q_{i}$, and public good provision, $x_{i}$. Let $p$ the relative price of the public good. Benefits from the public good are flow to neighbors, weighted by $\beta \in[0,1]$. Individual $i$ then maximizes $U_{i}=q_{i}^{\alpha}\left(x_{i}+\beta \sum_{i} g_{i j} x_{j}\right)^{1-\alpha}$ subject to the budget constraint $q_{i}+p x_{i} \leq w_{i}$. Simple computations yield the correspondence with $\bar{x}_{i}=\frac{1-\alpha}{p} w_{i}$ and $\delta=\alpha \beta$. Note that even if $\beta=1, \delta<1$. When own and neighbors' contributions are perfect substitutes in payoffs $U_{i}$, they are imperfect substitutes in the best reply function.
} 
the impact of others' play, captured in $\delta$, can vary across agents. Thus far, we have considered games where a agent $i$ 's best response is linear in the weighted sum of his neighbors' play, $\delta \sum_{j} g_{i j} x_{j}$. In many economic applications, the parameter $\delta$ may not be the same across all agents and may depend on an agent's graph position. For each agent $i$, we have $\delta_{i}(\mathbf{G})$ and $i$ 's linear best reply involves $\delta_{i}(\mathbf{G}) \cdot \sum_{j} g_{i j} x_{j}$. We can use our techniques to study any such game.

Consider, for example, settings where agents care about the average play of other players. Many games in microeconomics and macroeconomics - coordination games/beauty contests/social interactions games/investment games - have this feature. In many models, agents also have quadratic payoffs. ${ }^{52}$ While in some settings agents desire to coordinate their actions, other settings involve strategic substitutes, as when agents want to invest in different technologies. The standard treatments do not include networks, but a network treatment could transform the analysis. It would allow for social, geographic, and information structures and for mixes of strategic substitutes and complements. We call such models linear-in-means games. For each agent $i, \delta_{i}(\mathbf{G})=\delta \frac{1}{k_{i}}$, where $\delta$ is a constant and recall $k_{i}$ is the number of $i$ 's neighbors, and $i$ 's best reply is

$$
f_{i}(\mathbf{x})=\max \left(0,1-\delta \frac{1}{k_{i}}\left(\sum_{j=1}^{n} g_{i j} x_{j}\right)\right),
$$

with $\delta \in[0,1], g_{i j}=g_{j i} \in\{0,1\}$, and $\forall i, k_{i} \neq 0$. When $\mathbf{G}$ is not regular so that agents may have different numbers of neighbors, $\frac{1}{k_{i}} g_{i j} \neq \frac{1}{k_{j}} g_{j i}$, and the game with (modified) quadratic payoffs $\tilde{U}_{i}$ does not have a potential. But by appropriate rescaling of the payoffs, we obtain a "weighted potential," in the terminology of Monderer \& Shapley (1996). Define $\widetilde{\Pi}_{i}=k_{i} \tilde{U}_{i}$. The game with payoffs $\widetilde{\Pi}_{i}$ has the same best-replies and equilibria as the game with payoffs $\tilde{U}_{i}$. And we can easily see that $\frac{\partial^{2} \widetilde{\Pi}_{i i}(\mathbf{x})}{\partial x_{i} \partial x_{j}}=\frac{\partial^{2} \widetilde{\Pi}_{i}(\mathbf{x})}{\partial x_{j} \partial x_{i}}$ for all $i \neq j$. So this modified game has a potential function: $\varphi(\mathbf{x})=\sum_{i}\left(k_{i} x_{i}-\frac{1}{2} k_{i} x_{i}^{2}\right)-\frac{1}{2} \delta \sum_{j} g_{i j} x_{i} x_{j}$.

We derive conditions for unique and stable equilibria as follows: Introduce the network $\tilde{\mathbf{G}}$ such that $\tilde{g}_{i j}=\frac{g_{i j}}{\sqrt{k_{i}} \sqrt{k_{j}}}$. The potential is then strictly concave if and only if $\delta<-1 / \lambda_{\min }(\tilde{\mathbf{G}})$. The uniqueness condition now includes the absorptive capacity of the original graph "normalized" by agents' degrees. Similarly, the potential is not concave if and only if $\delta>-1 / \lambda_{\min }(\tilde{\mathbf{G}})$, and the results on multiple and corner equilibria apply. The stability results in Section IV all hold, again

\footnotetext{
${ }^{52}$ See discussions and models in, for example, Glaeser \& Scheinkman (2003) and Angeletos \& Pavan (2007).
} 
using $\tilde{\mathbf{G}}$.

As for comparative statics, observe that if $\mathbf{x}$ is an equilibrium, $\varphi(\mathbf{x})=\frac{1}{2} \sum_{i} k_{i} x_{i}$. Thus, comparing across global maxima, if $\delta$ increases, the sum of individual actions weighted by degrees decreases weakly. ${ }^{53}$ In contrast, the effect of adding links to the original graph $\mathbf{G}$ is not immediate. Connecting two agents increases their degrees which changes the weights used to compute the aggregate index. We show in Appendix that this new positive effect dominates the negative ones.

The rescaling procedure we describe works for any graph $g_{i j}=g_{j i} \in \mathbb{R}$ and payoff impacts $\delta_{i}(\mathbf{G})$ and it further indicates a class of directed graphs $\left(g_{i j} \neq g_{j i}\right)$ where our results apply. Consider a directed graph $\mathbf{H}$ and payoff impacts $\gamma_{i}$ such that for each $i$ and $j$ there are scalars $\alpha_{i}$ and $\alpha_{j}$ with the property $\alpha_{i} h_{i j}=\alpha_{j} h_{j i} .{ }^{54}$ We can define a graph $\mathbf{H}^{\prime}$ where for all $i$ and $j$, $h_{i j}^{\prime} \equiv \alpha_{i} h_{i j}=\alpha_{j} h_{j i} \equiv h_{j i}^{\prime}$. The analysis of a linear-best response game with graph $\mathbf{H}$ is then equivalent to that of a symmetric graph $\mathbf{H}^{\prime}$ and idiosyncratic payoff impacts $\delta_{i}=\gamma_{i} / \alpha_{i}$.

Strategic substitutes and complements. First, consider the basic model except $g_{i j} \in[-1,0]$ so that we have a game of pure strategic complements. ${ }^{55}$ The analysis of such games is relatively simple, as noted in Corbo et al. (2007). If $\delta<1 / \lambda_{\max }(-\mathbf{G})$ there is a unique interior equilibrium. With $x_{i} \in[0, \infty)$, an equilibrium fails to exist if $\delta>1 / \lambda_{\max }(-\mathbf{G})$ since complementarities have an explosive effect on actions. Since $\lambda_{\max }(-\mathbf{G})=-\lambda_{\min }(\mathbf{G})$, this result matches Proposition 2. Our results also show this unique, interior equilibrium (when it exists) is stable. Comparative statics are also straightforward. Following an increase in one agent $i$ 's action, $i$ 's neighbors increase their actions, then their neighbors increase theirs, and so on. Our analysis thus confirms what we already know, via a different route. ${ }^{56}$ However, these clear-cut results break down as soon as any substituability is present.

The question then is how to approach a game that is any mix of strategic substitutes and

\footnotetext{
${ }^{53}$ The similarity with the model with heterogeneous thresholds is not a coincidence. Apply the change of variables $y_{i}=x_{i} / \sqrt{k_{i}}$. Then $\widetilde{\Pi}_{i}=\left(k_{i}\right)^{3 / 2} y_{i}-\frac{1}{2} y_{i}^{2}-\delta \sum_{j} \tilde{g}_{i j} y_{i} y_{j}$. This corresponds to a model with thresholds $\bar{y}_{i}=\left(k_{i}\right)^{3 / 2}$. Then, $\sum_{i} \bar{y}_{i} y_{i}=\sum_{i} k_{i} x_{i}$. These thresholds, however, are determined by the network.

${ }^{54}$ This property holds if and only if in $\mathbf{H}$, for every triangle $i, j, k, h_{i j} \cdot h_{j k} \cdot h_{k i}=h_{i k} \cdot h_{k j} \cdot h_{j i}$; that is, for a triangle the product of bilateral links is the same clockwise and counterclockwise.

${ }^{55}$ There are strategic complementarities when for all $i j: g_{i j} \leq 0$ and $\delta \geq 0$, or $g_{i j} \geq$ and $\delta \leq 0$.

${ }^{56}$ The potential is strictly concave if and only if $\delta<1 / \lambda_{\max }(-\mathbf{G})$. Proposition 2 shows that uniqueness holds in that range. Because of the change of sign, Proposition 5 now says that aggregate action increases as complementarities increase.
} 
strategic complements; i.e., $g_{i j}=g_{j i} \in \mathbb{R}$. Because of complementarities, existence of a Nash equilibrium is not always guaranteed when $x_{i} \in[0, \infty)$. One fruitful approach is to bound the action space. With $x_{i} \in[0, l]$, we have best-reply

$$
f_{i}(\mathbf{x})=\min \left(l, \max \left(0,1-\delta \sum_{j=1}^{n} g_{i j} x_{j}\right)\right)
$$

An equilibrium exists for any $\delta$ and $\mathbf{G}$, and the potential and its properties are unchanged. However, we have new constraints on the problem $(\mathrm{P})$. Our uniqueness and stability results then apply with appropriate reformulation of boundary conditions.

Non-Linear Best Replies. Our analysis can be applied, locally, to any continuous potential game (see Appendix). Consider a game $\Gamma$ with $x_{i} \in \mathbb{R}_{+}$and payoff function $V_{i} \cdot{ }^{57}$ Construct a game $\widetilde{\Gamma}[\mathbf{x}]$ where the payoffs are given by the second-order Taylor approximations of the payoffs $V_{i}$ around $\mathbf{x}$. Then, best-replies of $\widetilde{\Gamma}[\mathbf{x}]$ are linear and approximate $\Gamma$ 's best-replies around $\mathbf{x}$; they capture the precise shape of strategic interactions around $\mathbf{x}$. Thus, $\mathbf{x}$ is a Nash equilibrium of $\Gamma$ if and only if it is a Nash equilibrium of $\widetilde{\Gamma}[\mathbf{x}]$. Furthermore, $\widetilde{\Gamma}[\mathbf{x}]$ is a potential game when $\Gamma$ is a potential game, in which case it can be analyzed using our techniques and results. The study of $\widetilde{\Gamma}[\mathbf{x}]$ may then provide relevant information for the study of $\Gamma$. In particular, because the Hessians of the potentials are the same, when $\mathbf{x}$ is stable in $\widetilde{\Gamma}[\mathbf{x}]$ it is stable in $\Gamma$ and the stability properties are usually equivalent. Future research, discussed next, will explore further such relationships.

\section{Conclusion: Future Research}

This paper brings a general network analysis to a wide class of games. A graph, or interaction matrix, gives whose actions directly affect whom. This matrix can represent, variously, social links, geography or market structure. We unify two strands of previous work and study games that have the same linear best replies. A canonical game in this class is one with quadaratic payoffs. Since all games in the class have the same best replies, they have the same equilibria. We derive equilibrium conditions for any graph and any level of payoff impact, using the theory

\footnotetext{
${ }^{57} \mathrm{As}$ in the rest of our analysis, $V_{i}$ is required to be twice continuously differentiable and $\partial^{2} V_{i} / \partial x_{i}^{2}<0$.
} 
of potential games and spectral graph theory.

We uncover a network measure that is fundamental to equilibrium analysis: the lowest eigenvalue. This eigenvalue captures what we call the absorptive capacity of the graph. The network is most absorptive when everyone is linked to everyone. It is least absorptive when it is complete bipartite, with as equal-sized sides as possible, and all agents are not linked to agents on their side but are linked to everyone on the other side. We find that when absorptive capacity is high, the graph contains the impact of agents' play on others, and there is a unique equilibrium. When absorptive capacity is low, payoff impacts are not contained by the graph, and there is always an equilibrium that involves extreme play. Except for highly absorptive graphs, stable equilibria are never symmetric or interior.

These results give a general intuition that outcomes are likely to be highly asymmetric and involve small sets of active agents. Only for very dense, highly clustered social structures are we likely to see equal distribution of efforts. The findings thus reinforce the notion of the "The Law of the Few" coined by Gladwell (2000) to describe the many cases he studies where a few people do most of the work or have a large influence. Bramoullé \& Kranton (2007) and Goyal \& Galeotti (2010) also emphasize such asymmetric outcomes. The present paper provides general results and shows that such asymmetries arise for wide range of payoff impacts and graph structures.

There are several directions for future work. Theoretical research could take two tacks. First, we will consider games where payoff functions do not yield linear best-replies. As discussed above, our analysis can be applied locally to any potential game. In addition, we can see that Lemmas 1 and 2 apply more generally; ${ }^{58}$ hence, this analysis is a stepping stone for a global analysis of a wider game class. A second theoretical tack is to study network formation. The analysis here is again a stepping stone: if agents could make and break links, any equilibrium of such a larger game is an equilibrium of the fixed-network game. ${ }^{59}$

The paper also provides predictions for empirical and experimental work. With small numbers of actors, the equilibrium set can be reasonably estimated for different graph structures and hypothesized payoff impact levels. For any given network, researchers can also calculate the lowest eigenvalue and use it either to test predictions or calibrate their models. The analysis

\footnotetext{
${ }^{58}$ Lemma 1 holds for any continuous potential game, and Lemma 2 holds for continuous potential games when equilibria are locally unique.

${ }^{59}$ That is, if an action vector and graph $(\mathbf{x}, \mathbf{G})$ is an equilibrium a network formation game, $\mathbf{x}$ must be an equilibrium of the game where individuals choose actions given $\mathbf{G}$. The same is true for stable equilibria.
} 
would indicate likely outcomes in geographic, social, or market settings, such as different product oligopoly, innovation, and experimentation with new technologies. ${ }^{60}$ Researchers conducting laboratory experiments (as in, e.g., Kearns, Suri \& Montfort (2006)) can also manipulate network structure and test if outcomes follow predicted patterns.

\footnotetext{
${ }^{60}$ Empirical studies of experimentation and local information spread in networks include Foster \& Rosenzweig's (1995) study of high-yield crop varieties in India and Conley \& Udry's (2008) study of pineapple farmers in Ghana.
} 


\section{APPENDIX}

\section{Proof of statements in Section II.}

The equilibrium set is a finite union of compact convex sets. Given a subset of agents $S$, define $E_{S}$ as the set of equilibria such that $\left(\mathbf{I}+\delta \mathbf{G}_{S}\right) \mathbf{x}_{S}=\mathbf{1}$ and $\forall i \notin S, x_{i}=0$. (The set of active agents, $A$, is included in $S$ but may be smaller). Let $E$ be the set of equilibria. Proposition 1 implies that $E=\cup_{S} E_{S}$. Next, show that $E_{S}$ is compact and convex. Note that $\mathbf{x} \in E_{S}$ if and only if $\left(\mathbf{I}+\delta \mathbf{G}_{S}\right) \mathbf{x}_{S}=\mathbf{1}, \forall i \notin S, x_{i}=0$ and $\delta \mathbf{G}_{N-S, S} \mathbf{x}_{S} \geq \mathbf{1}$. Given a converging sequence of elements of $E_{S}$, these three sets of conditions still hold when taking the limit. Thus, $E_{S}$ is closed hence compact. Second, let $\mathbf{x}, \mathbf{x}^{\prime} \in E_{S}, \sigma \in[0,1]$ and $\mathbf{x}^{(\sigma)}=\sigma \mathbf{x}+(1-\sigma) \mathbf{x}^{\prime}$. By linearity, $\left(\mathbf{I}+\delta \mathbf{G}_{S}\right) \mathbf{x}_{S}^{(\sigma)}=\mathbf{1}, \forall i \notin S, x_{i}^{(\sigma)}=0$ and $\delta \mathbf{G}_{N-S, S} \mathbf{x}_{S}^{(\sigma)} \geq \mathbf{1}$. Thus $\mathbf{x}^{(\sigma)} \in E_{S}$ and $E_{S}$ is convex. QED.

Individuals with a superset of neighbors take lower equilibrium action, for $\delta<1$. Suppose that $\delta<1$ and that $i$ is connected to $j$ and all $j$ 's neighbors. Suppose first that $x_{i}, x_{j}>0$. Then, $x_{i}+\delta\left(x_{j}+\sum_{k \neq i, j} g_{i k} x_{k}\right)=1$ and $x_{j}+\delta\left(x_{i}+\sum_{k \neq i, j} g_{j k} x_{k}\right)=1$. Subtracting both equalities yields $(1-\delta)\left(x_{i}-x_{j}\right)=-\sum_{k \neq i, j}\left(g_{i k}-g_{j k}\right) x_{k}$ hence $x_{i} \leq x_{j}$. Suppose next that $x_{j}=0$. Then $\delta\left(x_{i}+\sum_{k \neq i, j} g_{j k} x_{k}\right) \geq 1$ hence $\delta\left(x_{i}+\sum_{k \neq i, j} g_{i k} x_{k}\right) \geq 1$. If $x_{i}>0$, then $(1-\delta) x_{i}+\delta\left(x_{i}+\right.$ $\left.\sum_{k \neq i, j} g_{i k} x_{k}\right)=1$ which is impossible. Thus, $x_{i}=0$. QED.

\section{Proof of statements in Section III.}

The best-reply is contracting if and only if $\delta<1 / \rho(\mathbf{G})$. Introduce a vector $\mathbf{e}(\mathbf{x})=\mathbf{1}-\delta \mathbf{G x}$ such that $f_{i}(\mathbf{x})=\max \left(0, e_{i}(\mathbf{x})\right)$. We can see that $\left|f_{i}(\mathbf{x})-f_{i}\left(\mathbf{x}^{\prime}\right)\right| \leq\left|e_{i}(\mathbf{x})-e_{i}\left(\mathbf{x}^{\prime}\right)\right|$. For instance, if $f_{i}(\mathbf{x})>0$ and $f_{i}\left(\mathbf{x}^{\prime}\right)=0$, then $\left|f_{i}(\mathbf{x})-f_{i}\left(\mathbf{x}^{\prime}\right)\right|=f_{i}(\mathbf{x})$ while $\left|e_{i}(\mathbf{x})-e_{i}\left(\mathbf{x}^{\prime}\right)\right|=f_{i}(\mathbf{x})-e_{i}\left(\mathbf{x}^{\prime}\right)$ with $e_{i}\left(\mathbf{x}^{\prime}\right)<0$. Therefore, $\left\|\mathbf{f}(\mathbf{x})-\mathbf{f}\left(\mathbf{x}^{\prime}\right)\right\| \leq\left\|\mathbf{e}(\mathbf{x})-\mathbf{e}\left(\mathbf{x}^{\prime}\right)\right\|$ under the Euclidean norm. Next, $\mathbf{e}(\mathbf{x})-\mathbf{e}\left(\mathbf{x}^{\prime}\right)=-\delta \mathbf{G}\left(\mathbf{x}-\mathbf{x}^{\prime}\right)$, and $\left\|\mathbf{e}(\mathbf{x})-\mathbf{e}\left(\mathbf{x}^{\prime}\right)\right\|^{2}=\delta^{2}\left(\mathbf{x}-\mathbf{x}^{\prime}\right)^{T} \mathbf{G}^{2}\left(\mathbf{x}-\mathbf{x}^{\prime}\right)$. We then apply the Rayleigh-Ritz theorem, see Horn \& Johnson (1985, p. 176). Since $\lambda_{\max }\left(\delta^{2} \mathbf{G}^{2}\right)=\delta^{2} \rho(\mathbf{G})^{2}$, this yields: $\left\|\mathbf{e}(\mathbf{x})-\mathbf{e}\left(\mathbf{x}^{\prime}\right)\right\|^{2} \leq \delta^{2} \rho(\mathbf{G})^{2}\left\|\mathbf{x}-\mathbf{x}^{\prime}\right\|^{2}$. Thus, $\left\|\mathbf{f}(\mathbf{x})-\mathbf{f}\left(\mathbf{x}^{\prime}\right)\right\| \leq \delta \rho(\mathbf{G})\left\|\mathbf{x}-\mathbf{x}^{\prime}\right\|$ and $\mathbf{f}$ is contracting if $\delta \rho(\mathbf{G})<1$. Reciprocally, since $\mathbf{G}$ is nonnegative, it has a nonnegative eigenvector $\mathbf{x}_{0}$ associated to the eigenvalue $\rho(\mathbf{G})$, see Horn \& Johnson $\left(1985\right.$, p. 503). Let $\mathbf{x}=\lambda \mathbf{x}_{0}$ and $\mathbf{x}^{\prime}=\lambda^{\prime} \mathbf{x}_{0}$ with $\lambda, \lambda^{\prime}>0$. If $\lambda, \lambda^{\prime}$ are small enough, $\mathbf{f}(\mathbf{x})=\mathbf{e}(\mathbf{x})=\mathbf{1}-\delta \rho(\mathbf{G}) \mathbf{x}$ and the same holds for $\mathbf{f}\left(\mathbf{x}^{\prime}\right)$. Thus, $\mathbf{f}(\mathbf{x})-\mathbf{f}\left(\mathbf{x}^{\prime}\right)=\delta \rho(\mathbf{G})\left(\mathbf{x}-\mathbf{x}^{\prime}\right)$ and $\mathbf{f}$ is not contracting if $\delta \rho(\mathbf{G}) \geq 1$. QED. 
Proposition 2 gives a stronger uniqueness condition than previous conditions. Suppose that $-1 / \lambda_{\min }(\mathbf{G})<\underline{\delta}_{B C A Z}(\mathbf{G})$ and consider $\delta$ such that $-1 / \lambda_{\min }(\mathbf{G})<\delta<\underline{\delta}_{B C A Z}(\mathbf{G})$. Ballester, Calvó-Armengol \& Zenou (2006) show there is then a unique equilibrium for $\delta$ and $\mathbf{G}$ and this equilibrium is interior. By Proposition 3, there exists an equilibrium for $\delta$ and $\mathbf{G}$ which is a corner. This is a contradiction, hence $-1 / \lambda_{\min }(\mathbf{G}) \geq \underline{\delta}_{B C A Z}(\mathbf{G})$. Next, observe that for any graph $\mathbf{H}, k_{\min }(\mathbf{H}) \leq \lambda_{\max }(\mathbf{H}) \leq k_{\max }(\mathbf{H})$ (Cvetković et al. (1979)). This implies that $\lambda_{\max }(\mathbf{C}-\mathbf{G}) \geq k_{\min }(\mathbf{C}-\mathbf{G})=n-1-k_{\max }(\mathbf{G})$. Hence, $1 /\left(1+\lambda_{\max }(\mathbf{C}-\mathbf{G})\right) \leq 1 /\left(n-k_{\max }(\mathbf{G})\right)$. Then, $-\lambda_{\min }(\mathbf{G}) \leq \lambda_{\max }(\mathbf{G}) \leq k_{\max }(\mathbf{G})$, hence $-1 / \lambda_{\min }(\mathbf{G}) \geq 1 / k_{\max }(\mathbf{G})$ and $-1 / \lambda_{\min }(\mathbf{G})>$ $\underline{\delta}_{B C A Z}(\mathbf{G})$ if $k_{\max }(\mathbf{G})<n / 2$. QED.

Proof of Lemma 2. We first show that the potential increase along the trajectories of the system. Here, $\frac{d}{d t} \varphi(\mathbf{x}(t))=\sum_{i} \frac{\partial \varphi}{\partial x_{i}} \dot{x}_{i}=\nabla \varphi(\mathbf{x}) \cdot H(\mathbf{x})$.

Lemma A1. $\forall \mathbf{x} \in \mathbb{R}_{+}^{n}, \nabla \varphi(\mathbf{x}) \cdot H(\mathbf{x}) \geq 0$ and $\nabla \varphi(\mathbf{x}) \cdot H(\mathbf{x})=0$ if and only if $\mathbf{x}$ is a Nash equilibrium.

Proof. We have: $\frac{\partial \varphi}{\partial x_{i}}=1-\delta \sum_{j} g_{i j} x_{j}-x_{i}$ and $h_{i}(x)=1-\delta \sum_{j} g_{i j} x_{j}-x_{i}$ if $\delta \sum_{j} g_{i j} x_{j} \leq 1$ and $-x_{i}$ if $\delta \sum_{j} g_{i j} x_{j}>1$. Then, $\frac{\partial \varphi}{\partial x_{i}} h_{i}=\left(1-\delta \sum_{j} g_{i j} x_{j}-x_{i}\right)^{2}$ in the first case and $x_{i}\left(\delta \sum_{j} g_{i j} x_{j}-1+x_{i}\right)$ in the second case. Therefore, $\nabla \varphi(\mathbf{x}) \cdot H(\mathbf{x}) \geq 0$. Equality occurs iff $x_{i}=1-\delta \sum_{j} g_{i j} x_{j}$ if $\delta \sum_{j} g_{i j} x_{j} \leq 1$ and $x_{i}=0$ if $\delta \sum_{j} g_{i j} x_{j}>1$.

Next, consider a strategy profile $\mathbf{x}$. There are three possibilities. First, suppose that $\mathbf{x}$ is not a maximum of the potential. There is a sequence $\mathbf{x}^{m} \in \mathbb{R}_{+}^{n}$ converging to $\mathbf{x}$ such that $\varphi\left(\mathbf{x}^{m}\right)>\varphi(\mathbf{x})$. Since $\varphi$ cannot decrease along trajectories, starting at $\mathbf{x}^{m}$ the system cannot converge back to $\mathbf{x}$. Hence $\mathbf{x}$ is not asymptotically stable. Second, suppose that $\mathbf{x}$ is a maximum but is not locally strict. There is then a sequence $\mathbf{x}^{m}$ of potential maxima converging to $\mathbf{x}$ such that $\mathbf{x}^{m} \neq \mathbf{x}$ and $\varphi\left(\mathbf{x}^{m}\right)=\varphi(\mathbf{x})$. Since $\mathbf{x}^{m}$ is an equilibrium, it is a steady state of the system of differential equations. Thus, starting at $\mathbf{x}^{m}$ the system does not converge back to $\mathbf{x}$, and $\mathbf{x}$ is not asymptotically stable.

Third, suppose that $\mathbf{x}$ is a locally strict maximum of the potential. If there exists an $\varepsilon>0$ such that $\mathbf{x}$ is the unique equilibrium on $B_{O}(\mathbf{x}, \varepsilon) \cap \mathbb{R}_{+}^{n}$, then we can apply Theorem 6.4 in Weibull (1995). Precisely, the Lyapunov function is equal to $\varphi(\mathbf{x})-\varphi(\mathbf{y})$ for any $\mathbf{y} \in B_{O}(\mathbf{x}, \varepsilon) \cap \mathbb{R}_{+}^{n}$. Hence $\mathbf{x}$ is asymptotically stable. Thus, we only need to show that $\mathbf{x}$ is a locally strict maximum. Suppose the contrary. There exists a sequence of equilibria $\mathbf{x}^{m}$ converging to $\mathbf{x}$ such that $\mathbf{x}^{m}$ 
is not a potential maximum. Without loss of generality, we can assume that $\forall k, \mathbf{x}^{m}$ have the same active agents $A$ since there is a finite number of subsets of $N$. Hence $\left(\mathbf{I}+\delta \mathbf{G}_{A}\right) \mathbf{x}_{A}^{m}=\mathbf{1}$ and $\delta \mathbf{G}_{N-A, A}^{m} \mathbf{x}_{A} \geq 1$. Taking the limit shows that $\left(\mathbf{I}+\delta \mathbf{G}_{A}\right) \mathbf{x}_{A}=\mathbf{1}$ and $\delta \mathbf{G}_{N-A, A} \mathbf{x}_{A} \geq 1$, hence that any active agent of $\mathbf{x}$ is in $A$. Therefore, we can write $\mathbf{x}^{m}=\mathbf{x}+\mathbf{y}^{m}$ with $\mathbf{y}^{m}$ such that $\mathbf{y}_{N-A}^{m}=\mathbf{o}$ and $\left(\mathbf{I}+\delta \mathbf{G}_{A}\right) \mathbf{y}_{A}^{m}=\mathbf{o}$. Next, compute $\varphi\left(\mathbf{x}^{m}\right)$ in two different ways. First, $\varphi\left(\mathbf{x}^{m}\right)=\frac{1}{2}\left(\mathbf{x}^{m}\right)^{T} \mathbf{1}=\varphi(\mathbf{x})+\frac{1}{2}\left(\mathbf{y}^{m}\right)^{T} \mathbf{1}$ since $\mathbf{x}^{m}$ and $\mathbf{x}$ are equilibria. Second, by definition, $\varphi\left(\mathbf{x}^{m}\right)=\varphi\left(\mathbf{x}+\mathbf{y}^{m}\right)=\mathbf{x}^{T} \mathbf{1}+\left(\mathbf{y}^{m}\right)^{T} \mathbf{1}-\frac{1}{2}\left(\mathbf{x}+\mathbf{y}^{m}\right)^{T}(\mathbf{I}+\delta \mathbf{G})\left(\mathbf{x}+\mathbf{y}^{m}\right)$. The last expression is equal to $\left(\mathbf{x}_{A}+\mathbf{y}_{A}^{m}\right)^{T}\left(\mathbf{I}+\delta \mathbf{G}_{A}\right)\left(\mathbf{x}_{A}+\mathbf{y}_{A}^{m}\right)=\mathbf{x}_{A}^{T}\left(\mathbf{I}+\delta \mathbf{G}_{A}\right) \mathbf{x}_{A}$. Thus, $\varphi\left(\mathbf{x}^{m}\right)=\varphi(\mathbf{x})+\left(\mathbf{y}^{m}\right)^{T} \mathbf{1}$. This shows that $\left(\mathbf{y}^{m}\right)^{T} \mathbf{1}=\mathbf{o}$. Hence $\varphi\left(\mathbf{x}^{m}\right)=\varphi(\mathbf{x})$ which contradicts the fact that $\mathbf{x}$ is a locally strict maximum. QED.

Proof of Proposition 4. We analyze the second-order conditions of problem (P). Consider an equilibrium $\mathbf{x}$ with active agents $A$ and knife-edge inactive agents $K$, such that $A \cup K=\{i$ : $\left.x_{i}+\delta \sum_{j} g_{i j} x_{j}=1\right\}$ and $K=\left\{i: x_{i}=0\right.$ and $\left.\delta \sum_{j} g_{i j} x_{j}=1\right\}$. The application of Theorems 3.4 and 3.6 in Lee, Tam \& Yen (2005) to our setting yields the following result.

Lemma A2. Second-order conditions of problem (P).

$\mathbf{x}$ is a maximum of $\varphi$ on $\mathbb{R}_{+}^{n}$ iff $\forall \varepsilon \in \mathbb{R}^{A \cup K}$ such that $\varepsilon_{K} \geq \mathbf{o}, \varepsilon^{T}\left(\mathbf{I}+\delta \mathbf{G}_{A \cup K}\right) \varepsilon \geq 0$

$\mathbf{x}$ is a locally strict maximum of $\varphi$ on $\mathbb{R}_{+}^{n}$ iff $\forall \varepsilon \in \mathbb{R}^{A \cup K}-\{\mathbf{o}\}$ such that $\varepsilon_{K} \geq \mathbf{o}, \varepsilon^{T}(\mathbf{I}+$ $\left.\delta \mathbf{G}_{A \cup K}\right) \boldsymbol{\varepsilon}>0$

We derive necessary conditions. Observe that when $\varepsilon_{K}=\mathbf{o}, \boldsymbol{\varepsilon}^{T}\left(\mathbf{I}+\delta \mathbf{G}_{A \cup K}\right) \boldsymbol{\varepsilon}=\boldsymbol{\varepsilon}_{A}^{T}(\mathbf{I}+$ $\left.\delta \mathbf{G}_{A}\right) \varepsilon_{A}$. Therefore, if $\mathbf{x}$ is a maximum then $\mathbf{I}+\delta \mathbf{G}_{A}$ is positive semi-definite while if $\mathbf{x}$ is a locally strict maximum, $\mathbf{I}+\delta \mathbf{G}_{A}$ is positive definite. In contrast, observe that if $\mathbf{x}$ is a minimum of $\varphi$, then $\mathbf{I}+\delta \mathbf{G}_{A}$ must be negative semi-definite which is impossible. Therefore, any Nash equilibrium which is not a maximum of the potential is a saddle point.

When $K=\varnothing$, the second-order conditions take a simple form: $\mathbf{x}$ is a maximum iff $\mathbf{I}+\delta \mathbf{G}_{A}$ is positive semi-definite and it is a locally strict maximum iff $\mathbf{I}+\delta \mathbf{G}_{A}$ is positive definite. We next show that this situation is generic.

Lemma A3. Given a graph $\mathbf{G}$, for almost every $\delta$, there is no knife-edge agent in any equilibrium. Proof: Take a graph $\mathbf{G}$ such that $\mathbf{I}+\delta \mathbf{G}$ is invertible. Define $P_{i}(\delta)=\operatorname{det}(\mathbf{I}+\delta \mathbf{G})\left[(\mathbf{I}+\delta \mathbf{G})^{-1} \mathbf{1}\right]_{i}$. Consider the classic relation between a matrix's inverse and its cofactors (e.g. Horn \& Johnson (1985, p.20)). Let $m_{i j}$ be the determinant of the submatrix of $\mathbf{I}+\delta \mathbf{G}$ obtained by removing the 
$i$ th row and $j$ th column. Then, $P_{i}(\delta)=\sum_{j=1}^{n}(-1)^{i+j} m_{j i}$ hence $P_{i}$ is a polynomial of degree less than or equal to $n-1$ in $\delta$. In addition, $P_{i}(0)=1$ so $P_{i}$ cannot have more than $n-1$ zeros. Thus, for almost any $\delta$, the profile $\mathbf{y}=(\mathbf{I}+\delta \mathbf{G})^{-1} \mathbf{1}$ is well-defined and satisfies $\forall i, y_{i} \neq 0$. Next, for any equilibrium $\mathbf{x}, \mathbf{x}_{A \cup K}=\left(\mathbf{I}+\delta \mathbf{G}_{A \cup K}\right)^{-1} \mathbf{1}$ if $\operatorname{det}\left(\mathbf{I}+\delta \mathbf{G}_{A \cup K}\right) \neq 0$, hence for almost any $\delta$, $\forall i \in A \cup K, x_{i}>0$ and $K=\varnothing$.

\section{Proof of Statements in Section IV.}

The sets of active agents of stable equilibria are minimal for inclusion. Consider a stable equilibrium $\mathbf{x}$ with active agents $A$. For any $\mathbf{y} \in \mathbb{R}^{A}$, define $\varphi_{A}(\mathbf{y} ; \delta, \mathbf{G})=\varphi(\hat{\mathbf{y}} ; \delta, \mathbf{G})$ where $\hat{\mathbf{y}}$ is such that $\hat{\mathbf{y}}_{A}=\mathbf{y}$ and $\hat{\mathbf{y}}_{N-A}=\mathbf{o}$ and let $\left(P_{A}\right)$ be the problem: $\max _{\mathbf{y}} \varphi_{A}(\mathbf{y})$ under the constraints that $\forall i \in A, y_{i} \geq 0$. Since $\mathbf{x}$ is a locally strict maximum of $\varphi, \mathbf{I}+\delta \mathbf{G}_{A}$ is positive definite. Therefore, $\varphi_{A}$ is strictly concave and $\mathbf{x}_{A}$ is the unique solution to the Kuhn-Tucker conditions of $\left(P_{A}\right)$. If $\mathrm{x}^{\prime}$ is another equilibrium with active agents $A^{\prime} \subset A, \mathbf{x}_{A}^{\prime}$ must solve the Kuhn-Tucker conditions of $\left(P_{A}\right)$, which is impossible. QED.

Proof of Eigenvalue Results 1 and 2. Given its simplicity and independent interest, we reproduce here the proof provided to us by Noga Alon. By the Rayleigh-Ritz theorem (Horn \& Johnson (1985, p. 176)), $\lambda_{\min }(\mathbf{G})=\min _{\|\mathbf{x}\|=1} \mathbf{x}^{T} \mathbf{G x}$. Let $\mathbf{u}$ be an eigenvector associated with $\lambda_{\min }(\mathbf{G})$ and define $\mathbf{v}=\frac{1}{\|\mathbf{u}\|} \mathbf{u}$. Then, $\lambda_{\min }(\mathbf{G})=\mathbf{v}^{T} \mathbf{G} \mathbf{v}=\sum_{i, j \in R} g_{i j} v_{i} v_{j}+\sum_{i, j \in T} g_{i j} v_{i} v_{j}+$ $\sum_{i \in A, j \in B} g_{i j} v_{i} v_{j}$. Next, consider a graph $\mathbf{G}^{\prime}$ with less links within $R$ and $S$ and more links between $R$ and $S$. Observe that $v_{i} v_{j} \geq 0$ when $i, j \in R$ and $i, j \in S$, but $v_{i} v_{j} \leq 0$ when $i \in R, j \in S$. This means that $\mathbf{v}^{T} \mathbf{G}^{\prime} \mathbf{v} \leq \mathbf{v}^{T} \mathbf{G} \mathbf{v}$. Since $\lambda_{\min }\left(\mathbf{G}^{\prime}\right) \leq \mathbf{v}^{T} \mathbf{G}^{\prime} \mathbf{v}$, Result 2 follows.

Next, observe that we can cut all links within $R$ and within $S$ and add all links between $R$ and $S$. Hence $\lambda_{\min }$ is smallest for complete bipartite graphs. Then, if a complete bipartite graphs connects two groups of sizes $p$ and $n-p$, we know that $\lambda_{\min }=-\sqrt{p(n-p)}$ (Cvetkovic et al. (1979)), which is lowest when $p$ is equal to $n / 2$ when $n$ even and $(n-1) / 2$ when $n$ odd. QED.

\section{Proof of Statements in Section VI.}

Concentrating action on fewer agents increases overall play. Consider $\mathbf{x}$ and $\mathbf{x}^{\prime}$ two equilibria with active agents $A$ and $A^{\prime}$ such that $A \subset A^{\prime}$. Introduce $\mathbf{u}=(\mathbf{I}+\delta \mathbf{G}) \mathbf{x}$ and $\mathbf{u}^{\prime}=(\mathbf{I}+\delta \mathbf{G}) \mathbf{x}^{\prime}$. Observe that $\forall i, u_{i}, u_{i}^{\prime} \geq 1$. Compute $\mathbf{x}^{T}(\mathbf{I}+\delta \mathbf{G}) \mathbf{x}^{\prime}$ in two different ways. First, $\mathbf{x}^{T}(\mathbf{I}+\delta \mathbf{G}) \mathbf{x}^{\prime}=$ $\mathbf{x}^{T} \mathbf{u}^{\prime}=\sum_{i} u_{i}^{\prime} x_{i}$. Since $A \subset A^{\prime}, x_{i}>0 \Rightarrow x_{i}^{\prime}>0 \Rightarrow u_{i}^{\prime}=1$. Therefore, $\mathbf{x}^{T}(\mathbf{I}+\delta \mathbf{G}) \mathbf{x}^{\prime}=\sum_{i} x_{i}$. 
Second, $\mathbf{x}^{T}(\mathbf{I}+\delta \mathbf{G}) \mathbf{x}^{\prime}=[(\mathbf{I}+\delta \mathbf{G}) \mathbf{x}]^{T} \mathbf{x}^{\prime}=\mathbf{u}^{T} \mathbf{x}^{\prime}=\sum_{i} u_{i} x_{i}^{\prime} \geq \sum_{i} x_{i}^{\prime}$. QED.

Strict comparative statics. Suppose that $\delta^{\prime}>\delta, \mathbf{G}^{\prime}=\mathbf{G}$ and $\varphi\left(\mathbf{x} ; \delta^{\prime}, \mathbf{G}\right)$ and $\varphi(\mathbf{x} ; \delta, \mathbf{G})$ have the same maximum value over $\mathbb{R}_{+}^{n}$. Since $\varphi\left(\mathbf{x} ; \delta^{\prime}, \mathbf{G}\right) \leq \varphi(\mathbf{x} ; \delta, \mathbf{G})$, both functions have a common global maximum $\mathbf{x}^{*}$. This profile $\mathbf{x}^{*}$ is an equilibrium at $\delta$ and at $\delta^{\prime}$ and satisfies $\varphi\left(\mathbf{x}^{*} ; \delta^{\prime}, \mathbf{G}\right)=$ $\varphi\left(\mathbf{x}^{*} ; \delta, \mathbf{G}\right)$. Since $\varphi(\mathbf{x} ; \delta, \mathbf{G})=\mathbf{x}^{T} \mathbf{1}-\frac{1}{2} \mathbf{x}^{T}(\mathbf{I}+\delta \mathbf{G}) \mathbf{x}$, we have $\left(\mathbf{x}^{*}\right)^{T} \mathbf{G} \mathbf{x}^{*}=0$. Hence the active agents of $\mathbf{x}^{*}$ form an independent set of $\mathbf{G}$ and $\mathbf{x}^{*}$ is specialized. Next, suppose that $\delta^{\prime}=\delta$, $\mathbf{G} \neq \mathbf{G}^{\prime}$ and $\varphi(\mathbf{x} ; \delta, \mathbf{G})$ and $\varphi\left(\mathbf{x} ; \delta, \mathbf{G}^{\prime}\right)$ have the same maximum value over $\mathbb{R}_{+}^{n}$. Again, both functions have a common global maximum $\mathbf{x}^{*}$. It now satisfies $\left(\mathbf{x}^{*}\right)^{T}\left(\mathbf{G}^{\prime}-\mathbf{G}\right) \mathbf{x}^{*}=0$ and no link in $\mathbf{G}^{\prime}-\mathbf{G}$ connects two active agents of $\mathbf{x}^{*}$. QED.

For $\delta=1$, a specialized equilibrium associated with a largest maximal independent set is a global maximum of the potential. Suppose that $\delta=1$ and let $\mathbf{x}$ be an equilibrium with active agents $A$. For an agent $i$, let $N_{i}(A)$ be the set of $i$ 's neighbors in $A$. Then, $\forall i \in A, x_{i}+\sum_{j \in N_{i}(A)} x_{j}=1$. Take a maximal independent set $I$ of $\mathbf{G}_{A}$. Any agent in $A$ is either in $I$ or connected to an agent in I. Thus, $A=\bigcup_{i \in I}\{i\} \cup N_{i}(A)$. Therefore, $\sum_{i \in N} x_{i}=\sum_{i \in A} x_{i} \leq \sum_{i \in I}\left(x_{i}+\sum_{j \in N_{i}(A)} x_{j}\right)=|I|$. Next, observe that $I$ is an independent set of the graph $\mathbf{G}$, hence is included in a maximal independent set $I^{\prime}$. This yields: $\sum_{i \in N} x_{i} \leq\left|I^{\prime}\right|$ and total play in equilibrium is always lower than or equal to the size of the largest maximal independent set. QED.

Comparative statics on individual play in bipartite graphs. Suppose that $\mathbf{G}$ is connected and bipartite. Consider the model with heterogeneous thresholds. Let $\mathbf{x}$ be an interior equilibrium with $\delta<-1 / \lambda_{\min }(\mathbf{G})$. Then, for any $j$ on $i$ 's side, $\partial x_{j} / \partial \bar{x}_{i}>0$ while for any $j$ on the other side $\partial x_{j} / \partial \bar{x}_{i}<0$. To see why, note that $\mathbf{x}=(\mathbf{I}+\boldsymbol{\delta} \mathbf{G})^{-1} \overline{\mathbf{x}}$, hence $\partial \mathbf{x} / \partial \bar{x}_{i}=(\mathbf{I}+\boldsymbol{\delta} \mathbf{G})^{-1} \mathbf{u}_{i}$ where $u_{i i}=1$ and $u_{i j}=0$ if $j \neq i$. Since $\mathbf{G}$ is bipartite, $\lambda_{\min }(\mathbf{G})=-\rho(\mathbf{G})$ and we can write $(\mathbf{I}+\delta \mathbf{G})^{-1}=$ $\sum_{k=0}^{+\infty}(-\delta)^{k} \mathbf{G}^{k}$. Thus, $\partial x_{j} / \partial \bar{x}_{i}=\sum_{k=0}^{+\infty}(-\delta)^{k}\left(\mathbf{G}^{k}\right)_{j i}$ where $\left(\mathbf{G}^{k}\right)_{j i}=\sum_{l_{1}, \ldots, l_{k-1}} g_{j l_{1}} g_{l_{1} l_{2} \ldots} g_{l_{k-1} i}$ counts the number of paths of length $k$ connecting $j$ to $i$. On bipartite graphs, all cycles have even length. Any path is the union of a shortest path and of cycles. Therefore, if the shortest distance between $j$ and $i$ in $\mathbf{G}$ is even, all paths connecting them have even length. This happens when $j$ and $i$ are on the same side. Terms for $k$ odd in the sum are equal to zero and $\partial x_{j} / \partial \bar{x}_{i}>0$. Conversely, if the distance between them is odd they are on different sides and all paths connecting them have odd length. Terms for $k$ even are equal to zero and $\partial x_{j} / \partial \bar{x}_{i}<0$. Also, since the 
subgraph of a bipartite graph is bipartite, the result extends to any equilibrium $\mathbf{x}$ with active agents $A$ when $\delta<-1 / \lambda_{\min }\left(\mathbf{G}_{A}\right)$. And if $\mathbf{G}$ is not bipartite, it has an odd cycle and direct and indirect effects are not perfectly aligned. QED.

Proof of Proposition 6. Consider a stable equilibrium $\mathbf{x}$ with active agents $A$. Define $\varphi_{A}$ as above. Then, $\varphi_{A}$ is strictly concave and $\mathbf{x}_{A}$ is a global maximum of $\varphi_{A}$ on $\mathbb{R}_{+}^{A}$. Since $A^{\prime} \subset A$, $\frac{1}{2} \sum_{i \in N} x_{i}^{\prime}=\varphi_{A}\left(\mathbf{x}_{A}^{\prime} ; \delta^{\prime}, \mathbf{G}^{\prime}\right) \leq \varphi_{A}\left(\mathbf{x}_{A}^{\prime} ; \delta, \mathbf{G}\right) \leq \varphi_{A}\left(\mathbf{x}_{A} ; \delta, \mathbf{G}\right)=\frac{1}{2} \sum_{i \in N} x_{i}$. The first inequality comes from the monotonicity of the potential. The second inequality holds because $\mathbf{x}_{A}$ is a global maximum of $\varphi_{A}$. QED.

\section{Proof of statements in Section VII.}

Linear-in-means. Let $\mathbf{D}$ be the diagonal matrix such that $d_{i i}=k_{i}$. Then, the Hessian of the potential is $-(\mathbf{D}+\delta \mathbf{G})$. For any $\mathbf{x} \in \mathbb{R}^{n}$, define $\mathbf{y}$ such that $y_{i}=\sqrt{k_{i}} x_{i}$. We can see that $\mathbf{x}^{T}(\mathbf{D}+\delta \mathbf{G}) \mathbf{x}=\mathbf{y}^{T}(\mathbf{I}+\delta \tilde{\mathbf{G}}) \mathbf{y}$. Therefore, $\varphi$ is strictly concave iff $\mathbf{I}+\delta \tilde{\mathbf{G}}$ is positive definite. Next, consider $i$ and $j$ such that $g_{i j}=0$ and define $\mathbf{G}^{\prime}=\mathbf{G}+i j$. We have: $\varphi\left(\mathbf{x} ; \delta, \mathbf{G}^{\prime}\right)-\varphi(\mathbf{x} ; \delta, \mathbf{G})=$ $x_{i}+x_{j}-\frac{1}{2} x_{i}^{2}-\frac{1}{2} x_{j}^{2}-\delta x_{i} x_{j} \geq x_{i}+x_{j}-\frac{1}{2} x_{i}^{2}-\frac{1}{2} x_{j}^{2}-x_{i} x_{j}=\left(x_{i}+x_{j}\right)\left(1-\frac{1}{2}\left(x_{i}+x_{j}\right)\right)$. Since $x_{i}, x_{j} \leq 1, \varphi\left(\mathbf{x} ; \delta, \mathbf{G}^{\prime}\right) \geq \varphi(\mathbf{x} ; \delta, \mathbf{G})$ for any $\mathbf{x}$. QED.

Games with non-linear best-replies. Consider a game $\Gamma$ with strategies $\mathbf{x} \in \mathbb{R}_{+}^{n}$ and utilities $V_{i}$ which are twice continuously differentiable and satisfy $\partial^{2} V_{i} / \partial x_{i}^{2}<0$. Fix a profile $\mathbf{x}^{*}$ with active agents $A$, and introduce $\tilde{\Gamma}\left[\mathbf{x}^{*}\right]$ the game with same strategies and payoffs $\tilde{V}_{i}(\mathbf{x})=V_{i}\left(\mathbf{x}^{*}\right)+(\mathbf{x}-$ $\left.\mathbf{x}^{*}\right)^{T} \nabla V_{i}\left(\mathbf{x}^{*}\right)+\frac{1}{2}\left(\mathbf{x}-\mathbf{x}^{*}\right)^{T} \nabla^{2} V_{i}\left(\mathbf{x}^{*}\right)\left(\mathbf{x}-\mathbf{x}^{*}\right)$. Denote by $f_{i}$ the best-replies of $\tilde{\Gamma}\left[\mathbf{x}^{*}\right]$. We have: $f_{i}(\mathbf{x})=\max \left(0, \bar{x}_{i}-\sum_{j} g_{i j} x_{j}\right)$ with $\bar{x}_{i}=-\frac{\partial V_{i} / \partial x_{i}}{\partial^{2} V_{i} / \partial x_{i}^{2}}\left(\mathbf{x}^{*}\right)+x_{i}^{*}+\sum_{j} g_{i j} x_{j}^{*}$ and $g_{i j}=\frac{\partial^{2} V_{i} / \partial x_{i} \partial x_{j}}{\partial^{2} V_{i} / \partial x_{i}^{2}}\left(\mathbf{x}^{*}\right)$. This corresponds to the first-order Taylor approximation of $\Gamma$ 's best-replies. Then, $\mathbf{x}^{*}=\mathbf{f}\left(\mathbf{x}^{*}\right)$ iff $\partial V_{i} / \partial x_{i}\left(\mathbf{x}^{*}\right)=0$ when $x_{i}^{*}>0$ and $\partial V_{i} / \partial x_{i}\left(\mathbf{x}^{*}\right) \leq 0$ if $x_{i}^{*}=0$, which is equivalent to the fact that $\mathbf{x}^{*}$ is an equilibrium in $\Gamma$. Next, suppose that $\Gamma$ has a potential $\psi$. Observe that Lemma 1 directly extends. Equilibria of $\Gamma$ solve the Kuhn-Tucker conditions of problem $(\Psi): \max \psi(\mathbf{x})$ s.t. $\mathbf{x} \geq \mathbf{o}$. Since, $\partial^{2} \tilde{V}_{i} / \partial x_{i} \partial x_{j}(\mathbf{x})=\partial^{2} V_{i} / \partial x_{i} \partial x_{j}\left(\mathbf{x}^{*}\right), \tilde{\Gamma}\left[\mathbf{x}^{*}\right]$ also has a potential $\varphi$. And indeed, there exists $\boldsymbol{\alpha} \in \mathbb{R}^{n}$ such that $\forall i, j, \alpha_{i} g_{i j}=\alpha_{j} g_{j i}$, so our analysis of the linear-in-means case applies here. In particular, assume that $\mathbf{x}^{*}$ has no knife-edge inactive agents. Then, $\mathbf{x}^{*}$ is a locally strict maximum of $\varphi$ iff $\mathbf{G}_{A}$ is positive definite. The second-order conditions of problem $(\Psi)$ say that if $\mathbf{G}_{A}$ is positive definite, then $\mathbf{x}^{*}$ is a locally strict maximum of $\psi$ while if $\mathbf{x}^{*}$ is a 
locally strict maximum of $\psi$, then $\mathbf{G}_{A}$ is positive semi-definite. Finally, observe that Lemma 2 extends to general games when all equilibria are locally unique. In that case, barring knife-edge situations, $\mathbf{x}^{*}$ is stable in $\tilde{\Gamma}\left[\mathbf{x}^{*}\right]$ if and only if $\mathbf{x}^{*}$ is stable in $\Gamma$. QED. 


\section{REFERENCES}

Alon, Noga and Benny Sudakov. 2000. "Bipartite Subgraphs and the Smallest Eigenvalue," Combinatorics, Probability, and Computing, vol. 9, pp. 1-12.

Angeletos, George-Marios and Alessandro Pavan. 2007. "Efficient Use of Information and Social Value of Information," Econometrica 75 (4), pp. 1103-1142.

Ballester, Coralio, and Antoni Calvó-Armengol. 2007. "Moderate Interactions in Games with Induced Complementarities," mimeo, Universitat Autonoma de Barcelona.

Ballester, Coralio, Calvó-Armengol, Antoni, and Yves Zenou. 2006. "Who's who in networks. Wanted: the key player ," Econometrica, 74(5), 1403-1417.

Bell, Francis K., Cvetković, Dragoš, Rowlinson, Peter, and Slobodan K. Simić, 2008a. "Graphs for which the least eigenvalue is minimal, I" Linear Algebra and its Applications, 429, p. 234-241.

Bell, Francis K., Cvetković, Dragoš, Rowlinson, Peter, and Slobodan K. Simić, 2008b. "Graphs for which the least eigenvalue is minimal, II" Linear Algebra and its Applications, 429, p. 21682179

Bénabou, Roland. 2008. "Groupthink: Collective Delusions in Organizations and Markets," working paper.

Bergemann, Dirk and Stephen Morris. 2007. "Belief Free Incomplete Information Games," Discussion Paper No 1629, Cowles Foundation for Research in Economics, Yale University.

Bergemann, Dirk and Stephen Morris. 2008. "The Role of the Common Prior in Robust Implementation," Journal of the European Economic Association 6(2-3), pp. 551-559.

Bergemann, Dirk and Stephen Morris. 2009. "Robust Implementation in Direct Mechanisms," Review of Economic Studies, 76, pp. 1175-1204.

Bergstrom, Theodore, Blume, Lawrence, and Hal Varian. 1986. "On the Private Provision of Public Goods," Journal of Public Economics, 29, pp. 25-49.

Bloch, Francis and Unal Zenginobuz. 2007. "The Effect of Spillovers on the Provision of Local Public Goods," Review of Economic Design, 11, pp. 199-216.

Bollobas, Bela. 1998. Springer: Modern Graph Theory.

Bramoullé, Yann. 2007. "Anti-coordination and social interactions," Games and Economic Behavior, 58(1), 30-49.

Bramoullé, Yann, and Rachel Kranton. 2007. "Public Goods in Networks," Journal of Economic Theory, 135 (1), pp. 478-494.

Blume, Larry. 1993. "The statistical mechanics of strategic interaction," Games and Economic Behavior, 5(3), 387-424. 
Calvó-Armengol, Antoni, Patacchini, Eleanora and Yves Zenou. 2009. "Peer Effects and Social Networks in Education," Review of Economic Studies 76(4), pp. 1239-1267,

Calvó-Armengol, Antoni, and Yves Zenou. 2004. "Social Networks and Crime Decisions: The Role of Social Structure in Facilitating Delinquent Behavior," International Economic Review. vol 45, no 3, pp. 939-957.

Chien, S and A. Sinclair. 2007. "Convergence to approximate Nash equilibria in congestion games." In Proc. $18^{\text {th }}$ ACM-SIAM Symp. on Discrete Algorithms (SODA).

Conley, Timothy and Christopher Udry. 2008. "Learning about a New Technology: Pineapple in Ghana," forthcoming American Economic Review.

Constantine, Gregory. 1985. "Lower bounds on the spectra of symmetric matrices with nonnegative entries," Linear Algebra and its Applications, 65. pp.171-178.

Corbo, Jacomo, Calvó-Armengol, Antoni, and David Parkes. 2007. "The importance of network topology in local contribution games." In Proc. of the international workshop on Internet and Network Economics.

Cvetković, Dragos, Doob, Michael, and Horst Sachs. 1979. Spectra of Graphs, Theory and Applications, New York: Academic Press.

Desai, Madhav and Vasant Rao. 1994. "A Characterization of the Smallest Eigenvalue of a Graph," Journal of Graph Theory, vol. 18, no. 2, pp. 181-194.

Echenique, Frederico and Roland Fryer. 2007. "A Measure of Segregration based on Social Interactions," Quarterly Journal of Economics 122(2), pp. 441-485.

Fisher, Franklin M. 1961. "The Stability of the Cournot Oligopoly Solution: The Effects of Speeds of Adjustment and Increasing Marginal Costs," The Review of Economic Studies, Vol. 28, No. 2 Februrary, pp. 125-135

Foster, Andrew and Mark Rosenzweig. 1995. "Learning by Doing and Learning from Others: Human Capital and Technical Change," Journal of Political Economy, 103 (6), pp. 1176-1209.

Galeotti, Andrea, and Sanjeev Goyal. 2010. "Law of the Few," forthcoming American Economic Review.

Galeotti, Andrea, Goyal, Sanjeev, Jackson, Matthew, Vega-Redondo, Fernando, and Leeat Yariv. 2006. "Network Games," mimeo, Stanford University.

Galeotti, Andrea, Goyal, Sanjeev, Jackson, Matthew, Vega-Redondo, Fernando, and Leeat Yariv. 2009. "Network Games," Review of Economic Studies 77(1), pp. 218-244.

Gladwell, Malcolm. 2000. The Tipping Point. Boston: Little Brown.

Glaeser, Edward and José Scheinkman. 2003. "Non-Market Interactions," in Dewatripont, Hansen, and Turnovsky (eds.), Advances in Economics and Econometrics: Theory and Applications, Eighth World Congress, Volume I, Cambridge: Cambridge University Press, pp. 339-370. 
Goyal, Sanjeev and José Luis Moraga-Gonzáles. 2001. "R\&D Networks," Rand Journal of Economics, 32(4), pp. 686-707.

Grabher, G. 1993. The embedded firm: On the socioeconomics of industrial networks. London: Routledge.

Horn, Roger, and Charles, Johnson. 1985. Matrix Analysis. Cambridge: Cambridge University Press.

İlkiliç, Rahmi. 2008. "Network of Commons," working paper.

Kearns, Michael, Suri, Siddharth and Nick Montfort. 2006. "An experimental study of the coloring problem on human subject networks," Science 313(5788) pp. 824-827.

Lee, Gue Myung, Tam, Nguyen Nang, and Nguyen Dong Yen. 2005. Quadratic Programming and Affine Variational Inequalities. New York: Springer Science.

Monderer, Dov, and Lloyd Shapley. 1996. "Potential Games," Games and Economic Behavior, 14: 124-143.

Morris, Stephen and Takashi Ui. 2004. "Best Response Equivalence," Games and Economic Behavior, 49: 260-287.

Murty, K. and F.T. Yu. 1988. Linear Complementarity, Linear and non Linear Programming. Heldermann Verlag, Berlin.

Neyman, Abraham. 1997. "Correlated Equilibrium and Potential Games," International Journal of Game Theory, 26: 223-227.

Pardalos, Panos and Stephen Vavasis. 1991. "Quadratic Programming with One Negative Eigenvalue is NP-Hard," Journal of Global Optimisation, 1, pp. 15-22.

Roughgarden, T and É. Tardos. 2002. "How bad is selfish routing?," Journal of the ACM, 49 (2), pp. 236-259.

Scott, John. 2004. Social Network Analysis: A Handbook, Londong: Sage.

Trevisan, Luca. 2009. "Max Cut and the Smallest Eigenvalue," Proceedings of the 41st annual ACM symposium on Theory of computing, pp. 263-272

Vives, Xavier. 1999. Oligopoly Pricing: Old Ideas and New Tools, Cambridge: MIT Press.

Wasserman, Stanley and Katherine Faust. 1994. Social Network Analysis: Methods and Applications, Cambridge: Cambridge University Press.

Watts, Duncan J. and Steven H. Strogatz. 1998. "Collective dynamics of 'small-world' networks,". Nature 393, pp. 440-442.

Ye, Miao-Lin, Fan, Yi-Zheng, and Dong Liang, 2009. "The least eigenvalue of graphs with given connectivity," Linear Algebra and its Applications, 430, pp. 1375-1379. 
Young, Peyton. 1998. Individual Strategy and Social Structure, Princeton: Princeton University Press. 